\title{
Structural, Functional, and Metabolic Brain Differences as a Function of Gender Identity or Sexual Orientation: A Systematic Review of the Human Neuroimaging Literature
}

\author{
Alberto Frigerio $^{1}$ - Lucia Ballerini ${ }^{1}$ - Maria Valdés Hernández ${ }^{1}$ (D) \\ Received: 19 December 2018 / Revised: 6 April 2021 / Accepted: 8 April 2021 / Published online: 6 May 2021 \\ (c) The Author(s) 2021
}

\begin{abstract}
This review systematically explored structural, functional, and metabolic features of the cisgender brain compared with the transgender brain before hormonal treatment and the heterosexual brain compared to the homosexual brain from the analysis of the neuroimaging literature up to 2018, and identified and discussed subsequent studies published up to March 2021. Our main aim was to help identifying neuroradiological brain features that have been related to human sexuality to contribute to the understanding of the biological elements involved in gender identity and sexual orientation. We analyzed 39 studies on gender identity and 24 on sexual orientation. Our results suggest that some neuroanatomical, neurophysiological, and neurometabolic features in transgender individuals resemble those of their experienced gender despite the majority resembling those from their natal sex. In homosexual individuals the majority resemble those of their same-sex heterosexual population rather than their opposite-sex heterosexual population. However, it is always difficult to interpret findings with noninvasive neuroimaging. Given the gross nature of these measures, it is possible that more differences too subtle to measure with available tools yet contributing to gender identity and sexual orientation could be found. Conflicting results contributed to the difficulty of identifying specific brain features which consistently differ between cisgender and transgender or between heterosexual and homosexual groups. The small number of studies, the small-to-moderate sample size of each study, and the heterogeneity of the investigations made it impossible to meta-analyze all the data extracted. Further studies are necessary to increase the understanding of the neurological substrates of human sexuality.
\end{abstract}

Keywords Gender identity $\cdot$ Neuroimaging $\cdot$ Sexual orientation $\cdot$ Transgender $\cdot$ Transsexual

\section{Introduction}

\section{Sex, Gender Identity, and Sexual Orientation}

Human sexuality is a complex and multilevel structure made up of different components, and it is usually described by different perspectives and using different terminologies. Despite the terms sex and gender being used interchangeably, we refer "sex" to the biological condition (chromosomal, gonadal,

Supplementary Information The online version contains supplementary material available at https://doi.org/10.1007/ s10508-021-02005-9.

Maria Valdés Hernández

M.Valdes-Hernan@ed.ac.uk

1 Division of Health Sciences, University of Edinburgh, Edinburgh EH16 4SB, UK and phenotypic), "gender" to the inner psychological perception of one's own identity (gender identity) and to the outer cultural perception in behavior and habits attributed to and assumed by masculinity and femininity (gender role), and "sexual orientation" to sexual attraction (sexual preference) (Shah et al., 2012).

The search for the origin of gender identity and sexual orientation is part of the debate on the impact of nature and culture on human life (Lippa, 2002). This topic is highly controversial, due to its cultural, social, and political implications, and it is widely debated within the scientific community. Despite the efforts of scientists in conducting objective research, research on social problems are influenced by the cultural environment, and often reflect the dominant theories of their time (Jordan-Young, 2010). The vexata quaestio is: To what extent are gender and sexual orientation biologically determined and/or socially constructed by personal experiences and cultural expectations? 
On the one hand, the "born that way theory" holds that gender identity and sexual orientation are innate and fixed properties (Savic et al., 2010; Swaab, 2007, 2008). In this sense, brains of transgender and homosexual individuals would differ from brains of cisgenders and heterosexuals (Burke et al., 2017) in areas related to body perception (Savic \& Arver, 2011) and sexual arousal (Sylva, 2013). In this framework, some authors refer to an early programming of gender identity and sexual inclinations due to alterations in sexual differentiation in the brain, decoupled from genital differentiation, as in transgenderism, or reduced, as in homosexuality. They propose that such altered sexual differentiation in the brain causes an alteration in the development of the brain areas modulating body perception (in transgenderism) or sexual arousal (in homosexuality) (Burke et al., 2017). On the other hand, the so-called gender theory holds that gender identity and sexual orientation are just cultural constructions, and it denies any kind of biological influence (Butler, 1990).

Actually, both gender identity and sexual orientation seem to develop under two main types of influence: biological (genes, hormones, and gene expression) and environmental (influences of parents, peers, partners, and social models) factors (Altinay \& Anand, 2020; Balthazar, 2016; Jorge, 2010) as a result of the interaction between nature and culture (Hines, 2004). Evidence seems to suggest that biology contributes significantly to the development of both gender identity and sexual orientation (Roselli, 2018). Nevertheless, the idea that human sexuality is not biologically fixed is supported by longitudinal studies, which reported a certain fluidity in both gender identity (Drummond et al., 2008) and sexual orientation (Savin-Williams and Ream, 2007). Eventually, research on gender identity and sexual orientation is difficult because of the specificity of human sexuality, which makes difficult the use of animal models. Unlike animals, humans express their gender identity (Herbert, 2008), and their sexual behavior is, then, influenced by personal and social experiences and expectations (Maney, 2016).

\section{Studies on Gender Identity and Sexual Orientation}

To verify whether and which biological factors are involved in the development of gender identity and sexual orientation, several studies have been conducted comparing cisgenders vs. transgenders, and heterosexuals vs. homosexuals. These studies allow the verification of whether or not there are specific features which could be related to the development of transgender identity and same-sex attraction as opposed to cisgender identity and heterosexual orientation. In this case, they would allow researchers to infer which elements are involved in the development of gender identity and sexual orientation as a whole. But let us first clarify the relevant terminology.
While the term cisgender refers to people whose sense of gender identity corresponds to their birth sex, the term transgender refers to individuals who identify themselves with the gender opposite to that assigned at birth. If transgenders ask for a hormonal and/or surgical affirmation, they are called transsexuals (APA, 2013). While the term heterosexual refers to people who are emotionally, romantically or sexually attracted to people of the opposite sex, the term homosexual refers to people who feel an emotional, romantic or sexual attraction toward subjects of the same sex (APA, 2008).

It is also important to distinguish between early-onset vs. late-onset transgenderism. While early-onset transgenderism appears before puberty (i.e., during infancy), late-onset transgenderism appears after puberty (i.e., adolescence or adult age). The early-onset form has been reported associated with a same-sex sexual orientation and referred to androphilia in birth-assigned males and gynephilia in birth-assigned females; while in the late-onset form, which is more common in birth-assigned males than in birth-assigned females, heterosexual orientation is not uncommon (Lawrence, 2010).

The neural bases of gender identity and sexual orientation have been studied through neural, hormonal, and genetic investigations. Post mortem studies reported brain differences between cisgender and transgender people (GarciaFalgueras \& Swaab, 2008; Kruijver et al., 2000; Zhou et al., 1995) and between heterosexual and homosexual subjects (Allen \& Gorski, 1992; LeVay, 1991; Swaab \& Hofman, 1990). Hormonal research suggests the involvement of prenatal hormones in the development of transgender identity (Cohen-Kettenis, 2005; Dessens et al., 2005) and homosexual orientation (McFadden, 2002; Zucker et al., 1996). Genetic investigations suggest a possible hereditary component for transgenderism (Green, 2000; Heylens et al., 2012; Segal, 2006; Veale et al., 2010) and homosexuality (Drabant et al., 2012; Wijchers \& Festenstein, 2011). Overall, biological factors seem to play a role in shaping both gender identity and sexual orientation. Nevertheless, no evidence allows experts to conclude that they are determined by any specific factor, and many scientists think that both biological and social factors are involved in the development of gender identity (APA, 2014) and sexual orientation (APA, 2008).

A new frontier in research is represented by the neuroimaging techniques. A recent study shows pubertal testosterone being related to structural properties of the cerebral cortex (Liao et al., 2021). Imaging approaches focused on socioemotional processing, executive functioning, and self-concept/image domains have been also recommended to study neurodevelopmental effects in transgenderism (Chen et al., 2020). With regard to gender identity, four studies have nonsystematically discussed the results offered by neuroimaging. It has been pointed out that, before hormonal treatment, in transgenders the most important brain parameters, namely intracranial, gray matter, white matter, and cerebrospinal 
volumes, tend to be congruent with the gender assigned at birth - after hormone treatment they partly adjust to the characteristics of the desired gender -, although some structural, functional, and metabolic brain features may exhibit signs of masculinization or feminization (Guillamon et al., 2016; Kreukels \& Guillamon, 2016; Mueller et al., 2017; Smith et al., 2015). With regard to sexual orientation, the neuroimaging literature is scarce. Investigations have reported structural (Abé et al., 2014; Manzouri \& Savic, 2018; Ponseti et al., 2007; Savic \& Lindström, 2008; Witelson et al., 2008), functional (Hu et al., 2008, 2011, 2013, 2014; Kagerer et al., 2011; Manzouri \& Savic, 2018; Paul et al., 2008; Perry et al., 2013; Ponseti et al., 2009; Safron et al., 2017, 2018; Sylva, 2013; Zeki \& Romaya, 2010; Zhang \& Meaney, 2010), and metabolic (Berglund et al., 2006; Kinnunen et al., 2004; Savic \& Lindström, 2008; Savic et al., 2005) differences between heterosexual and homosexual individuals, but an attempt to summarize and analyze these reports is, to the best of our knowledge, nonexistent.

Overall, neuroimaging investigations on both gender identity and sexual orientation have reported conflicting results, with considerable overlap between transgender or homosexual people and control population. The lack of systematically extracted data limits the progress in these areas of research. We conducted a systematic review and meta-analysis to investigate whether or not there are structural, functional, and metabolic neuroimaging features that differentiate cisgender from transgender and heterosexual from homosexual individuals, in an attempt to provide the scientific community data gathered from the whole body of scientific literature that has been produced up to date, extracted and uniformly processed.

\section{Aim}

To document the scientific evidence from neuroimaging techniques on brain features that might be distinctive in cisgenders compared to transgenders (gender identity investigation), and in heterosexuals compared to homosexuals (sexual orientation investigation).

\section{Method}

\section{Systematic Literature Search}

A literature search was systematically conducted according to PRISMA (preferred reporting items for systematic reviews and meta-analyses) guidelines (Liberati, 2009). The search strategy, conducted in three different databases (Embase, Medline, PsycInfo), included articles published up to January 2018 comparing cisgenders vs. transgenders and articles published up to April 2018 comparing heterosexuals vs. homosexuals. Further, the initial search was updated with articles indexed in Medline from April 2018 up to March 2021.

Search terms used for the comparison between cisgenders and transgenders were brain AND (transgender OR transsexual OR gender dysphoria) AND (magnetic resonance imaging OR MRI OR diffusion tensor imaging OR DTI OR voxel-based morphometry OR VBM OR functional emission tomography OR fMRI OR positron emission tomography OR PET OR single photon emission computer tomography OR SPECT). Search terms used for the comparison between heterosexuals and homosexuals were brain AND (homosexual OR gay OR lesbian) AND (magnetic resonance imaging OR MRI OR diffusion tensor imaging OR DTI OR voxel-based morphometry OR VBM OR functional emission tomography OR fMRI OR positron emission tomography OR PET OR single photon emission computer tomography OR SPECT).

We analyzed only articles written in English and which published primary research output. Primary selection used title and abstract information. Authors were contacted if articles were not available online and/or if there was a question about the data presented in the article. After the initial selection, articles were checked for inclusion/exclusion criteria, and references were checked for possible further inclusions.

\section{Selection Criteria}

\section{Inclusion Criteria}

The analysis of gender identity included articles which compared cisgender (non-transgender) population (male control $=\mathrm{MC}$; female control $=\mathrm{FC}$ ) with transgender people (male-to-female $=\mathrm{MtF}$; female-to-male $=\mathrm{FtM}$ ) before hormonal treatment, while the analysis of the sexual orientation included articles which compared heterosexual people (heterosexual $\operatorname{man}=\mathrm{HeM}$; heterosexual woman $=\mathrm{HeW}$ ) with homosexual subjects (homosexual $\mathrm{man}=\mathrm{HoM}$; homosexual woman $=\mathrm{HoW}$ ).

\section{Exclusion Criteria}

Articles that investigated people affected by neurological diseases or by diseases associated with neurological outcome (e.g., HIV) were not included. As hormonal treatment may affect brain features (Rametti et al., 2012), studies and/or data on transsexuality after hormonal treatment were excluded.

\section{Data Extraction}

All quantitative outcomes including effect size and level of significance, regardless of whether or not they represented significant differences or not, were extracted from all papers included in the primary search conducted up to 2018. In 
addition, we independently extracted sample size, subject characteristics, mean age of subject population, type of neuroimaging technique (including field strength in the case of MRI), and regions of interest (ROI). Stereotaxic coordinates of activated/relevant brain areas were extracted from studies that used fMRI (resting state or not) and voxel-based morphometry (VBM). The findings from the papers identified in the period from April 2018 to March 2021 were summarized at the end of each correspondent subsection but not considered for the meta-analyses.

\section{Data Analysis}

Microsoft Excel 2016 was used to represent the distribution of the demographic data and imaging modalities from all studies. The data extracted from each ROI were tabulated and visualized to draw conclusions. GingerAle 2.3.6 software was used to meta-analyze the stereotaxic coordinates that showed relevance to our research question for those studies that provided this information (i.e., those that used fMRI and VBM).

Due to the low number of studies conducted with metabolic neuroimaging techniques (i.e., PET and SPECT), it was not possible to carry out a meta-analysis of the brain regions that could metabolically differ between the groups of individuals involved in both of the analyses. The number of studies that used brain structural MRI to explore brain characteristics in relation to sexual orientation was also reduced, not allowing to meta-analyze these data either. Instead, we summarized this information.

To calculate the risk of bias within and across studies, we used the Quadas tool (Whiting et al., 2003). Quantitative results were converted to OR and CI using Practical MetaAnalysis Effect Size Calculator by Wilson (http://www. campbellcollaboration.org/escalc/html/EffectSizeCalcu lator-Home.php). After extracting all data available, it was not possible to do a meta-analysis per brain area due to the low number of studies with numerical data (see http://dx.doi. org/10.7488/ds/2412).

\section{Results}

\section{Primary Literature Search}

The systematic search up to 2018 generated 492 publications from the three different databases: 268 for the analyses of gender identity and 224 for the analyses on sexual orientation. A total of 51 studies were included: 30 for the analyses of gender identity and 21 for the analyses on sexual orientation (Fig. 1 and Appendices 1 and 2). All studies were conducted using different neuroimaging techniques: structural (MRI), functional (fMRI and rs-fMRI), and metabolic (PET and SPECT) (Fig. 2). The majority of the studies included used functional MRI (28/51 studies: $61 \%$ of the studies on sexual orientation and $45 \%$ of the studies on gender identity). Studies that used PET and SPECT modalities were few in both analyses (13\% of the studies on gender identity and $18 \%$ of the studies on sexual orientation). Figure 3 shows the mean age and sample size of the groups of individuals involved in the analyses. The analysis on gender identity involved individuals across a wider age range (mean ages 9.5-46.7 years old, i.e., including two studies on gender identity in children in pre-puberty age (9-10 years old) and three in adolescents (14-16 years old)) than the analysis on sexual orientation (mean ages 22.1-33.4 years old), but none of them covered early infancy and neither later adulthood. Due to the small number of studies included and the heterogeneity in the information available (i.e., from the included studies), we did not classify the papers with respect to the homogeneity of the groups (i.e., in terms of social background, education, comorbidities, genetic and risk factors of the individuals involved).

\section{Gender Identity Analyses: Study Selection}

From 268 publications, 99 papers were duplicate or overlapped, 29 papers matched the inclusion criteria, and 140 were excluded. An additional study was included from the references (see Table 1 for study information).

\section{Sexual Orientation Analyses. Study Selection}

From 224 publications, 94 papers were duplicate or overlapped, 19 papers matched the inclusion criteria, and 111 were excluded. Two additional studies were included (see Table 2 for study information).

\section{Update from April 2018 until March 2021}

From 86 papers identified from the literature in this short period, $9 / 74$ publications in gender identity and $3 / 12$ publications on sexual orientation matched our inclusion/exclusion criteria.

\section{Regions of Interest Analyses}

\section{Gender Identity}

Structural MRI was conducted in 13 of the studies published up to 2018 . However, only one of them conducted the analysis in specific stereotaxic coordinates (Simon et al., 2013). The 12 studies that conducted ROI analysis involved 229 FtM, $169 \mathrm{MtF}, 478$ FC, and 484 MC. Table 3 shows the ROI and the parameters investigated by each of these studies. Two studies involving 79 out of $229 \mathrm{FtM}, 37$ out of $169 \mathrm{MtF}, 64$ out of $478 \mathrm{FtM}$, and 57 out of $484 \mathrm{MtF}$ did not find differences in the mean diffusivity of the hypothalamus (Kranz 


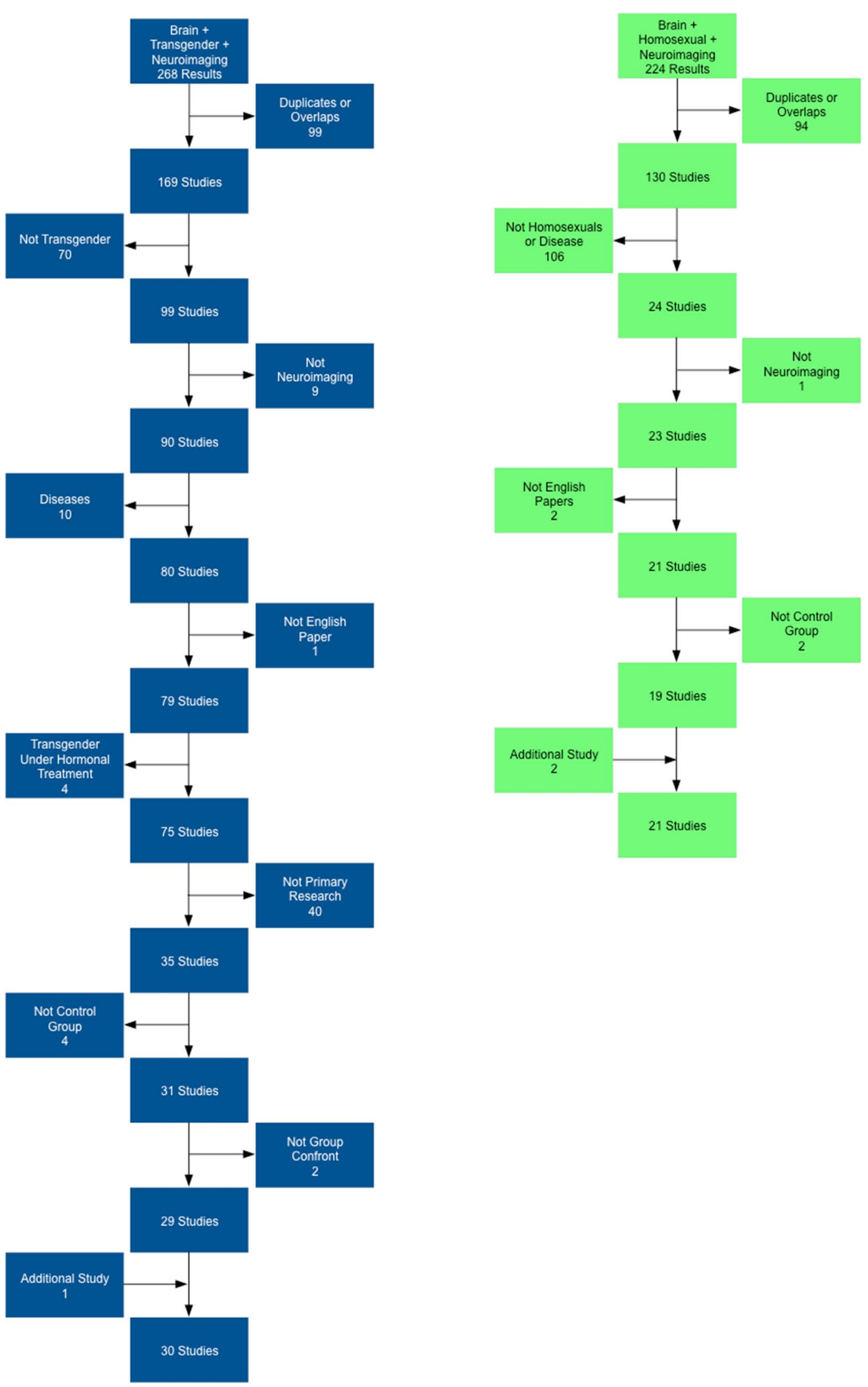

Fig. 1 Flowcharts summarizing the study selection process for the analyses on gender identity (left) and sexual orientation (right) 
Neuroimaging modalities of the studies included
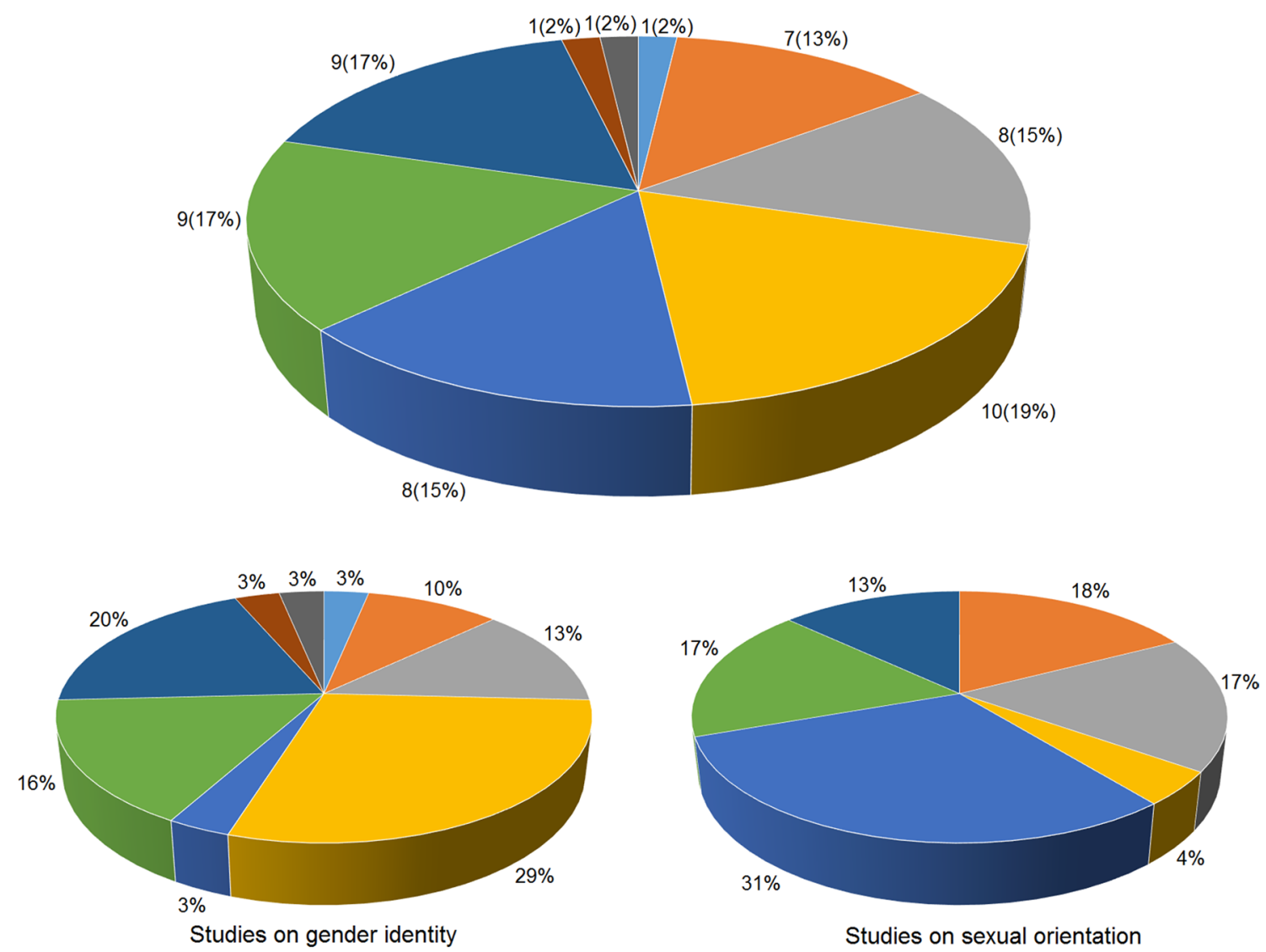

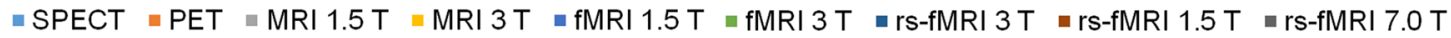

Fig. 2 3D pie charts summarizing the number and percentage of studies included in the analyses, conducted with different neuroimaging techniques: overall information (top), analysis on gender identity (bottom left), and analysis on sexual orientation (bottom right)

et al., 2018) nor in the volumes of cerebellum, hypothalamus, and medial frontal cortex (Hoekzema et al., 2015). Differences between cisgenders and transgenders were reported in 10/12 studies in white matter microstructure (four studies), volumetric analysis (four studies), cortical thickness (two studies), and corpus callosum shape (one study).

White matter microstructure of cisgender and transgender groups was analyzed by four studies. Only one study (23 FtM, $21 \mathrm{MtF}, 25 \mathrm{FC}$, and $25 \mathrm{MC}$ ) investigated the structural connectome, which is the complete map of the neural connections in a brain, and found differences between cisgender and transgender population in the right subcortical hemispheric connectivity ratio (Hahn et al., 2015). Three studies analyzed fractional anisotropy (FA) and mean diffusivity (MD), of which only one involving $18 \mathrm{FtM}, 19 \mathrm{FC}$, and $24 \mathrm{MC}$ found that in FtM FA was masculinized in some brain areas (right superior longitudinal fasciculus and in the forceps minor) and fell halfway between male and female patterns in other brain areas (corticospinal tract) (Rametti et al., 2011a). The other two studies either did not find significant differences in FA between different groups (Kranz et al., 2014b) (involved $23 \mathrm{FtM}, 21 \mathrm{MtF}, 23 \mathrm{FC}$, and $22 \mathrm{MC}$ ) or found that MtFs FA fell halfway between MC and FC pattern in some brain regions (right superior longitudinal fasciculus, forceps minor, corticospinal tract, right anterior cingulum) (Rametti et al., 2011b) (involved $18 \mathrm{MtF}, 19 \mathrm{MC}, 19 \mathrm{FC}$ ). MD in transgender groups (either MtF or FtM) was found to fall halfway between FC and MC people in corticospinal tract right and left and in forceps minor by one study that involved $23 \mathrm{FtM}, 21 \mathrm{MtF}$, 23 FC, 22 MC (Kranz et al., 2014b).

Subcortical gray matter volume was investigated by four studies. All of them found that the volume of the putamen was consistently different between cisgender and transgender groups (Luders et al., 2009; Manzouri et al., 2017; Savic \& Arver, 2011; Zubiaurre-Elorza, et al., 2013). Manzouri et al. (2017) found that the FtMs left putamen was larger than both female and male cisgenders (sample: 28 FtM, 34 FC, 34 MC), and Savic and Arver (2011) found that among 
Mean age and sample size

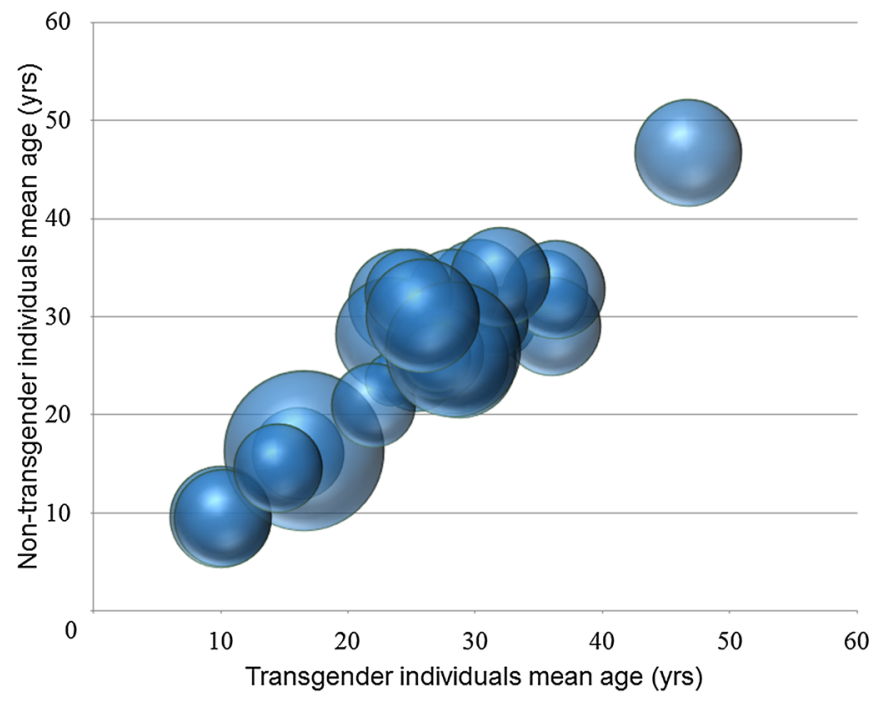

Fig. 3 3D bubble charts of the mean age and sample size of: a cisgenders and transgenders involved in the selected studies (left) (Note: the graph does not include the study by Yokota et al., 2005 because of the lack of data), and $\mathbf{b}$ heterosexuals and homosexuals involved in the selected studies (right) (Note: the graph does not include the study

all subcortical structures, MtF's putamen and thalamus were smaller than those in both female and male cisgender groups (sample: $24 \mathrm{MtF}, 24 \mathrm{MC}, 24 \mathrm{FC}$ ). These two studies also found that total gray matter volume did not differ between the transgender and cisgender groups. Zubiaurre-Elorza et al. (2013) also investigated subcortical gray matter in $24 \mathrm{FtM}$, $18 \mathrm{MtF}, 23 \mathrm{FC}$ and $29 \mathrm{MC}$ and reported that FtM had atypically larger right putamen, compared to the typical size of this brain structure in FC in average. Luders et al. (2009) investigated gray matter volumes in 22 different regions, 12 in the right hemisphere, and 10 in the left hemisphere (i.e., frontal, occipital and parietal lobes, superior frontal gyrus, midline, frontal pole, basal ganglia-caudate nucleus and putamen -, limbic system - subcallosum gyrus, mammillary body, amygdala, thalamus, hypothalamus, basal surface), in $24 \mathrm{MtF}, 30 \mathrm{MC}, 30 \mathrm{FC}$, and found putaminal volume in $\mathrm{MtF}$ to be atypically smaller (i.e., compared to the typical average putaminal volume in MC).

Cortical thickness was investigated in two studies, which reported differences between cisgender and transgender groups in only a few non-overlapping regions. One (28 FtM, 34 FC, 34 MC) found differences between FtM and both FC and $\mathrm{MC}$ in the supramarginal, parietal, rostral middle frontal, inferior temporal gyrus, superior frontal gyrus, and lingualprecalcarine cortex cuneus (Manzouri et al., 2017), and the other (24 FtM, $18 \mathrm{MtF}, 23 \mathrm{FC}, 29 \mathrm{MC}$ ) reported that MtFs had orbitofrontal, medial occipital and insular regions that resemble those typically seen in the female control group (Zubiaurre-Elorza et al., 2013). Manzouri and Savic, in

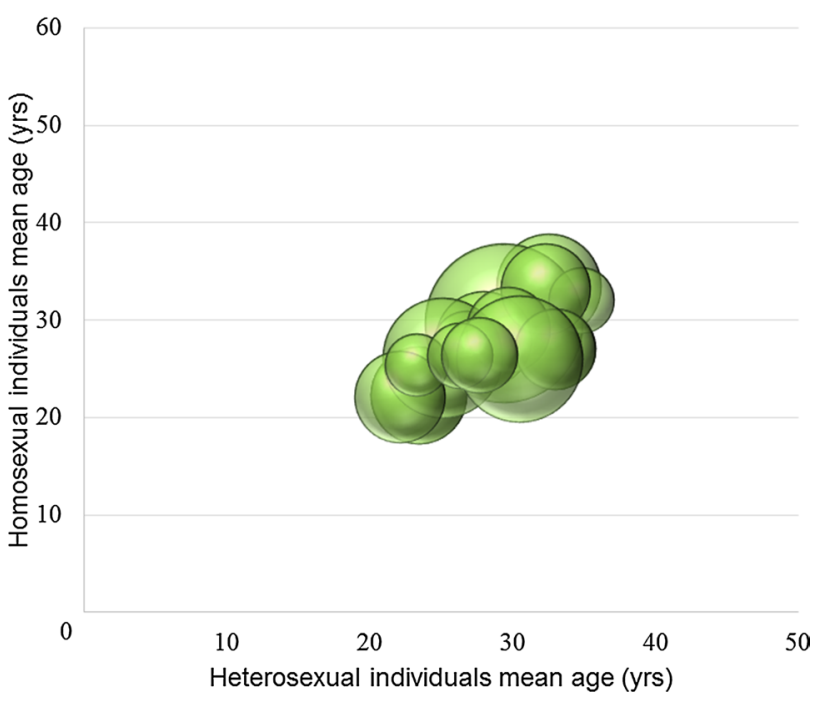

by $\mathrm{Hu}$ et al., 2011 because of the lack of data. Four studies (Kagerer et al., 2011; Perry et al., 2013; Sylva, 2013; Zeki \& Romaya, 2010) reported just the mean age of all the sample size, and we assumed that it was the same in heterosexual and homosexual subsamples)

another study published in 2019 involving $27 \mathrm{FtM}, 40 \mathrm{MtF}$, 40 heterosexual MC, 40 heterosexual FC, 30 homosexual $\mathrm{MC}$ and 30 homosexual FC found that cortical thickness did not differ between heterosexual controls and transgenders of the same birth-assigned sex. Transgender groups presented thicker clusters at the temporal and parietal cortex compared to heterosexual controls of their experienced gender, but these results were no longer observed when compared against homosexual controls. (Manzouri \& Savic, 2019).

Corpus callosum shape was investigated by only one study (28 FtM, $22 \mathrm{MtF}, 211 \mathrm{FC}, 211 \mathrm{MC}$ ). It found that in transgender people it was closer to their experienced gender than to their assigned sex at birth (Yokota et al., 2005).

Four additional studies analyzing structural brain differences between cisgenders and transgenders were identified in the period between April 2018 and March 2021. A study published in 2020 involving 26 males and females aged 19-38 concludes that the nucleus accumbens, left thalamus, right hippocampus, and right caudate nucleus were smaller in transgenders (sample size 11) than in cisgenders (sample size 15). However, did not specify the biological sex of the transgender and cisgender samples (Starcevic et al., 2020). In a large study, also published in the same year involving 121 individuals (mean ages: 27.17 (23 MtF), 30.17 (29 FtM), 27.09 (34 MC), 26.29 (35 FC) years old) authors manifest impossibility to conclude whether the brain structure of the transgender groups resemble or not the morphology of their respective gender identity (Baldinger-Melich et al., 2020). This result is not surprising given that all sample groups 
Table 1 Studies included in the analysis of gender identity

\begin{tabular}{|c|c|c|c|c|}
\hline $\begin{array}{l}\text { Study: First author's surname (if } \\
\text { one or more than two authors) }\end{array}$ & Year & Sample & Mean age & Technique \\
\hline Berglund & 2008 & $12 \mathrm{MtF}-12 \mathrm{MC}-12 \mathrm{FC}$ & $32-26-33$ & PET \\
\hline Burke & 2014 & $17 \mathrm{FtM}-19 \mathrm{MtF}-19 \mathrm{FC}-20 \mathrm{MC}$ & $9.6-10.4-9.7-9.5$ & fMRI \\
\hline Burke & 2016 & $21 \mathrm{FtM}-21 \mathrm{FC}-20 \mathrm{MC}$ & $16.1-16.3-15.9$ & fMRI \\
\hline Clemens & 2017 & $15 \mathrm{MtF}-21 \mathrm{MC}-20 \mathrm{FC}$ & $35.5-32.32-32.5$ & rs-fMRI \\
\hline Feusner & 2017 & 27 FtM-27 FC-27 MC & $24.2-32.1-31$ & rs-fMRI \\
\hline Gizewski & 2009 & $12 \mathrm{MtF}-12 \mathrm{MC}-12 \mathrm{FC}$ & $36-29-29$ & fMRI \\
\hline Hahn & 2015 & $23 \mathrm{FtM}-21 \mathrm{MtF}-25 \mathrm{FC}-25 \mathrm{MC}$ & $26.9-30.9-25.3-25.6$ & MRI \\
\hline Hoekzema & 2015 & $54 \mathrm{FtM}-37 \mathrm{MtF}-52 \mathrm{FC}-44 \mathrm{MC}$ & $16.92-16.05-16.29-16.42$ & MRI \\
\hline Junger & 2014 & $16 \mathrm{MtF}-21 \mathrm{MC}-20 \mathrm{FC}$ & $36.38-32.35-33.16$ & fMRI \\
\hline Kranz & $2014 \mathrm{a}$ & $14 \mathrm{MtF}-13 \mathrm{MC}-9 \mathrm{FC}$ & $31.4-29.8-29$ & PET \\
\hline Kranz & $2014 b$ & $23 \mathrm{FtM}-21 \mathrm{MtF}-23 \mathrm{FC}-22 \mathrm{MC}$ & $25.91-30.86-25.96-25.45$ & MRI \\
\hline Kranz & 2015 & $14 \mathrm{FtM}-19 \mathrm{MtF}-11 \mathrm{FC}-24 \mathrm{MC}$ & $28.21-31.79-30.43-34.14$ & PET \\
\hline Kranz & 2018 & 25 FtM-12 FC-13 MC & $27.24-24.42-28.77$ & MRI \\
\hline $\mathrm{Ku}$ & 2013 & 12 FtM-11 MtF-12 FC-11 MC & All Trans 25.4-All Cis 24.4 & rs-fMRI \\
\hline Lin & 2014 & $12 \mathrm{FtM}-11 \mathrm{MtF}-12 \mathrm{FC}-11 \mathrm{MC}$ & All Trans 25.4-All Cis 24.4 & rs-fMRI \\
\hline Luders & 2009 & $24 \mathrm{MtF}-30 \mathrm{MC}-30 \mathrm{FC}$ & $46.73-46.57-46.77$ & MRI \\
\hline Manzouri & 2017 & $28 \mathrm{FtM}-34 \mathrm{FC}-34 \mathrm{MC}$ & $23.5-27.6-28.8$ & MRI and rs-fMRI \\
\hline Nawata & 2010 & 11 FtM-9 FC & $23.4-23.6$ & SPECT \\
\hline Nota & 2017 & 13 FtM-18 MtF-18 FC-21 MC & $9.7-10.5-9.6-9.4$ & rs-fMRI \\
\hline Pol & 2006 & $6 \mathrm{FtM}-8 \mathrm{MtF}-6 \mathrm{FC}-9 \mathrm{MC}$ & $28-25-23-25$ & MRI \\
\hline Rametti & $2011 \mathrm{a}$ & 18 FtM-19 FC-24 MC & $28.24-31.22-33$ & MRI \\
\hline Rametti & $2011 b$ & $18 \mathrm{MtF}-19 \mathrm{MC}-19 \mathrm{FC}$ & 24.74-31.94-33 & MRI \\
\hline Santarnecchi & 2012 & $1 \mathrm{FtM}-25 \mathrm{FC}-25 \mathrm{MC}$ & $22-21-21$ & rs-fMRI \\
\hline Savic and Arver & 2011 & $24 \mathrm{MtF}-24 \mathrm{MC}-24 \mathrm{FC}$ & $32-33-35$ & MRI \\
\hline Schöning & 2010 & $11 \mathrm{MtF}-11 \mathrm{MC}$ & $37.55-33.09$ & fMRI \\
\hline Simon & 2013 & 7 FtM-10 MtF-7 FC-11 MC & $24.8-28.5-23.9-27.1$ & MRI \\
\hline Soleman & 2013 & $11 \mathrm{FtM}-6 \mathrm{MtF}-26 \mathrm{FC}-24 \mathrm{MC}$ & $14.64-14.25-14.44-14.68$ & fMRI \\
\hline Spies & 2016 & $33 \mathrm{FtM}-24 \mathrm{MtF}-44 \mathrm{FC}-33 \mathrm{MC}$ & $26.79-30.25-26.16-27.48$ & rs-fMRI \\
\hline Yokota & 2005 & $28 \mathrm{FtM}-22 \mathrm{MtF}-211 \mathrm{FC}-211 \mathrm{MC}$ & Not reported & MRI \\
\hline Zubiaurre-Elorza & 2013 & $24 \mathrm{FtM}-18 \mathrm{MtF}-23 \mathrm{FC}-29 \mathrm{MC}$ & $26.21-25.5-31.09-29.28$ & MRI \\
\hline
\end{tabular}

FtM, female-to male; MtF, male-to-female; FC, female control; MC, male control

involved non-balanced subsamples of individuals with different sexual orientation (i.e., heterosexuals, bisexuals and homosexuals). Two additional studies, one also published in 2020 involving 80 transgender and 60 cisgender non-western individuals (Khorashad et al., 2020), and another published in 2021 involving $16 \mathrm{FtM}, 17 \mathrm{FC}$ and 14 MC (Skorska et al., 2021) concluded that cortical morphometry (mainly surface area) was related to sex assigned at birth, but not to the experienced gender.

\section{Sexual Orientation}

Five MRI studies were analyzed (1/5 did not report the ROI analysis, but it provided stereotaxic coordinates using voxelbased morphometry). The four studies that conducted the
ROI analysis involved $81 \mathrm{HoM}$, $50 \mathrm{HoW}, 96 \mathrm{HeM}$, and 86 HeW.

Due to the low number of studies conducted with MRI, it was not possible to do a meta-analysis on structural features in homosexual subjects compared to heterosexual subjects. However, findings contained in these studies offer data worth describing. Table 4 shows the ROI and the parameters investigated by each of these studies.

Cortical thickness (CTh) was investigated by two studies (Abé et al., 2014; Manzouri \& Savic, 2018). While Abé et al. (2014) found that HoM have a thinner CTh than HeM in the visual area, Manzouri and Savic (2018) found that HoM have a thicker CTh than HeM in the parietal lobe. No significant differences were found between HoW and HeW.

Subcortical volumes were investigated by three studies (Abé et al., 2014; Manzouri \& Savic, 2018; Witelson et al., 
Table 2 Studies included in the analysis of sexual orientation

\begin{tabular}{|c|c|c|c|c|}
\hline Study reference & Year & Sample & Mean Age & Technique \\
\hline Abé et al & 2014 & 19 HoM-21 HeM-21 HeW & $33.5-31.9-33.2$ & MRI \\
\hline Berglund et al & 2006 & $12 \mathrm{HoW}-12 \mathrm{HeW}-12 \mathrm{HeM}$ & $33-26-28$ & PET \\
\hline Hu et al & 2013 & $26 \mathrm{HoM}-26 \mathrm{HeM}$ & $22.27-23.46$ & rs-fMRI \\
\hline Hu et al & 2014 & 26 HoM-26 HeM & $22.27-23.46$ & rs-fMRI \\
\hline Hu et al & 2011 & 14 HoM-14 HeM & Not reported & fMRI \\
\hline Hu et al & 2008 & 10 HoM-10 HeM & $26.5-27.9$ & fMRI \\
\hline Kagerer et al & 2011 & 11 HoM-10 HeM & All sample 28 & fMRI \\
\hline Kinnunen et al & 2004 & $8 \mathrm{HoM}-7 \mathrm{HeM}$ & $29-28$ & PET \\
\hline Manzouri-Savic & 2018 & $30 \mathrm{HoM}-30 \mathrm{HoW}-40 \mathrm{HeM}-40 \mathrm{HeW}$ & $31.4-27.9-29.5-29.3$ & $\begin{array}{l}\text { MRI and rs- } \\
\text { fMRI }\end{array}$ \\
\hline Paul et al & 2008 & 12 HoM-12 HeM & $32-34.8$ & fMRI \\
\hline Perry et al & 2013 & 12 HoM-12 HoW-13 HeM-15 HeW & All sample 28.46 & fMRI \\
\hline Ponseti et al & 2009 & 14 HoM-12 НеM & $27.4-26.8$ & fMRI \\
\hline ponseti et al & 2007 & 16 HoM-15 HoW-24 HeM-25 HeW & $27.3-24.9-25.3-24.9$ & MRI [VBM] \\
\hline Safron et al & 2017 & $\begin{array}{l}22 \mathrm{HoM}-23 \mathrm{HeM} \\
22 \mathrm{HoM}-19 \mathrm{HeM}\end{array}$ & $\begin{array}{l}33.2-32.3 \\
\text { Not reported }\end{array}$ & $\begin{array}{l}\text { fMRI [picture] } \\
\text { fMRI [video] }\end{array}$ \\
\hline Safron et al & 2018 & $\begin{array}{l}20 \mathrm{HoW}-20 \mathrm{HeW} \\
20 \mathrm{HoW}-18 \mathrm{HeW}\end{array}$ & $\begin{array}{l}29-29.7 \\
\text { Not reported }\end{array}$ & $\begin{array}{l}\text { fMRI [picture] } \\
\text { fMRI [video] }\end{array}$ \\
\hline Savic et al & 2005 & 12 HoM-12 HeM-12 HeW & $33-28-26$ & PET \\
\hline Savic-Lindström & 2008 & $\begin{array}{l}20 \text { HoM-20 HoW-25 HeM-25 HeW } \\
12 \text { HoM-12 HoW-13 HeM-13 HeW }\end{array}$ & $\begin{array}{l}32-20-30-31 \\
\text { Not reported }\end{array}$ & $\begin{array}{l}\text { MRI } \\
\text { PET }\end{array}$ \\
\hline Sylva et al & 2013 & 12 HoM-11 HoW-12 HeM-11 HeW & All sample 22.1 & fMRI \\
\hline Witelson et al & 2008 & 12 HoM-10 HeM & $25.4-23.3$ & MRI \\
\hline Zeki-Romaya & 2010 & 6 HoM-6 HoW-6 HeM-6 HeW & All sample 26.3 & fMRI \\
\hline Zhang et al & 2011 & 16 HoM-16 HeM & $26.4-27.7$ & fMRI \\
\hline
\end{tabular}

HoM, homosexual men; HoW, homosexual women; HeM, heterosexual men; $\mathrm{HeW}$, heterosexual women
2008). Abé et al. (2014) found a smaller thalamus volume in HoM than HeM, while Witelson et al. (2008) found that HoM had a larger corpus callosum in the isthmus region. No other significant effects of sexual orientation were found.

A study measured cerebral and cerebellar hemispheres (Savic \& Lindström, 2008). With regard to the cerebral hemisphere, they were symmetrical in HoM and in HeW, while they were asymmetrical in HoW and in HeM. With regards to the cerebellar hemisphere, no group had asymmetry. Another study investigated white matter tracts of the whole brain (Manzouri \& Savic, 2018), and did not find significant differences between heterosexual and homosexual groups.

A diffusion tensor imaging study published in 2020 involving 53 homosexual and 47 heterosexual men found lower connectivity between left postcentral gyrus and left supramarginal gyrus in the homosexual group compared to the heterosexual group (Wang, Hu, et al., 2020).

\section{Stereotaxic Coordinates Analysis}

\section{Gender Identity}

Six fMRI, eight rs-fMRI, and three VBM studies published up to 2018 were considered for meta-analyses. fMRI studies were conducted under visual stimulation (2), smelling stimulation (1), vocal stimulation (1), a mental rotation task (1), and a verbal fluency test (1). The 17 studies that conducted stereotaxic coordinates analysis involved $195 \mathrm{FtM}, 208 \mathrm{MtF}$, 347 FC, and 346 MC. Figure 4 displays six representative slices showing the foci resultant from the meta-analysis carried out using GingerAle 2.3.6 software using data from 12/17 studies (Burke et al., 2014, 2016; Clemens et al., 2017; Feusner et al., 2017; Gizewski et al., 2009; Hoekzema et al., 2015; Junger et al., 2014; Ku et al, 2013; Manzouri et al., 2017; Santarnecchi et al., 2012; Savic and Arver, 2011; Schöning et al., 2010; Simon et al, 2013) (see Appendix 3 in Supplementary Materials for the labels of each foci and Table 5 for the number of foci related to different brain areas). The meta-analyses conducted ("Transgender_vs_Cisgender Natal Sex", "Transgender_vs_Cisgender Opposite Sex”, and "Transgender_vs_Cisgender") showed that transgender people's brain activation differed more frequently in the Brodmann Areas (BA) 18 and 19, which include the occipital visual area along with BA 17 , which is involved in visual processing.

Five studies (1/6 fMRI and 4/8 rs-fMRI) did not report the stereotaxic coordinates, and and it was impossible to determine them in all but one case. A study (11 MtF, 12 
Table 3 Regions of Interest analysis on gender identity

\begin{tabular}{|c|c|c|c|c|c|c|}
\hline Reference & ROI & Parameters & Trans_vs_Natal Sex & Trans_vs_Opposite Sex & Trans_vs_Cis & M_vs_F \\
\hline \multirow[t]{4}{*}{ Hahn et al. (2015) } & Subcortical L & HCR & $\mathrm{Nr}$ & $\mathrm{Nr}$ & $\mathrm{Nd}$ & $\mathrm{Nd}$ \\
\hline & Subcortical R & HCR & $\mathrm{Nr}$ & $\mathrm{Nr}$ & $\mathrm{v}$ & $\mathrm{v}$ \\
\hline & Subcortical R-frontal R & LCW & $\mathrm{Nr}$ & $\mathrm{Nr}$ & $\mathrm{Nd}$ & $\mathrm{Nd}$ \\
\hline & Subcortical L-parietal L & LCW & $\mathrm{Nr}$ & $\mathrm{Nr}$ & $\mathrm{Nd}$ & $\mathrm{Nd}$ \\
\hline \multirow[t]{4}{*}{ Hoekzema et al. (2015) } & Cerebellum L & Volume & $\mathrm{Nd}$ & $\mathrm{Nr}$ & $\mathrm{Nr}$ & $\mathrm{v}$ \\
\hline & Cerebellum R & Volume & $\mathrm{Nd}$ & $\mathrm{Nr}$ & $\mathrm{Nr}$ & $\mathrm{v}$ \\
\hline & Hypothalamus & Volume & $\mathrm{x}$ & $\mathrm{Nr}$ & $\mathrm{Nr}$ & $\mathrm{v}$ \\
\hline & Medial frontal cortex & Volume & $\mathrm{Nd}$ & $\mathrm{Nr}$ & $\mathrm{Nr}$ & $\mathrm{v}$ \\
\hline Kranz et al. (2018) & Hypothalamus & MD & $\mathrm{x}$ & $\mathrm{x}$ & $\mathrm{x}$ & $\mathrm{v}$ \\
\hline \multirow[t]{12}{*}{ Kranz et al. (2014b) } & GM & Volume & $\mathrm{x}(\mathrm{MtF})-\mathrm{x}(\mathrm{FtM})$ & $\mathrm{v}(\mathrm{MtF})-\mathrm{v}(\mathrm{FtM})$ & $\mathrm{x}$ & $\mathrm{v}$ \\
\hline & WM & Volume & $\mathrm{x}(\mathrm{MtF})-\mathrm{x}(\mathrm{FtM})$ & $\mathrm{v}(\mathrm{MtF})-\mathrm{v}(\mathrm{FtM})$ & $\mathrm{x}$ & $\mathrm{v}$ \\
\hline & CSF & Volume & $\mathrm{x}(\mathrm{MtF})-\mathrm{x}(\mathrm{FtM})$ & $\mathrm{v}(\mathrm{MtF})-\mathrm{v}(\mathrm{FtM})$ & $\mathrm{x}$ & $\mathrm{v}$ \\
\hline & TIV & Volume & $\mathrm{x}(\mathrm{MtF})-\mathrm{x}(\mathrm{FtM})$ & $\mathrm{v}(\mathrm{MtF})-\mathrm{v}(\mathrm{FtM})$ & $\mathrm{x}$ & $\mathrm{v}$ \\
\hline & CST R & MD & $\mathrm{v}(\mathrm{MtF})-\mathrm{v}(\mathrm{FtM})$ & $\mathrm{v}(\mathrm{MtF})-\mathrm{v}(\mathrm{FtM})$ & $\mathrm{v}$ & $\mathrm{v}$ \\
\hline & CST L & MD & $\mathrm{v}(\mathrm{MtF})-\mathrm{v}(\mathrm{FtM})$ & $\mathrm{v}(\mathrm{MtF})-\mathrm{v}(\mathrm{FtM})$ & $\mathrm{v}$ & $\mathrm{v}$ \\
\hline & Forceps major & MD & $\mathrm{x}(\mathrm{MtF})-\mathrm{x}(\mathrm{FtM})$ & $\mathrm{x}(\mathrm{MtF})-\mathrm{x}(\mathrm{FtM})$ & $\mathrm{x}$ & $\mathrm{x}$ \\
\hline & Forceps minor & MD & $\mathrm{v}(\mathrm{MtF})-\mathrm{v}(\mathrm{FtM})$ & $\mathrm{v}(\mathrm{MtF})-\mathrm{v}(\mathrm{FtM})$ & $\mathrm{v}$ & $\mathrm{v}$ \\
\hline & CST R & FA & $\mathrm{x}(\mathrm{MtF})-\mathrm{x}(\mathrm{FtM})$ & $\mathrm{x}(\mathrm{MtF})-\mathrm{x}(\mathrm{FtM})$ & $\mathrm{x}$ & $\mathrm{x}$ \\
\hline & CST L & FA & $\mathrm{x}(\mathrm{MtF})-\mathrm{x}(\mathrm{FtM})$ & $\mathrm{x}(\mathrm{MtF})-\mathrm{x}(\mathrm{FtM})$ & $\mathrm{x}$ & $\mathrm{x}$ \\
\hline & Forceps major & FA & $\mathrm{x}(\mathrm{MtF})-\mathrm{x}(\mathrm{FtM})$ & $\mathrm{x}(\mathrm{MtF})-\mathrm{x}(\mathrm{FtM})$ & $\mathrm{x}$ & $\mathrm{x}$ \\
\hline & Forceps minor & FA & $\mathrm{v}(\mathrm{MtF})-\mathrm{v}(\mathrm{FtM})$ & $\mathrm{v}(\mathrm{MtF})-\mathrm{v}(\mathrm{FtM})$ & $\mathrm{x}$ & $\mathrm{x}$ \\
\hline \multirow[t]{14}{*}{ Luders et al. (2009) } & Frontal lobe & Volume & $\mathrm{x}$ & $\mathrm{v}$ & $\mathrm{Nd}$ & $\mathrm{v}$ \\
\hline & Occipital lobe & Volume & $\mathrm{x}$ & $\mathrm{v}$ & $\mathrm{Nd}$ & $\mathrm{v}$ \\
\hline & Parietal lobe & Volume & $\mathrm{x}$ & $\mathrm{v}$ & $\mathrm{Nd}$ & $\mathrm{v}$ \\
\hline & SFG & Volume & $\mathrm{x}$ & $\mathrm{v}$ & $\mathrm{Nd}$ & $\mathrm{v}$ \\
\hline & Midline & Volume & $\mathrm{x}$ & $\mathrm{v}$ & $\mathrm{Nd}$ & $\mathrm{v}$ \\
\hline & Frontal pole & Volume & $\mathrm{x}$ & $\mathrm{v}$ & $\mathrm{Nd}$ & $\mathrm{v}$ \\
\hline & Caudate nucleus & Volume & $\mathrm{x}$ & $\mathrm{v}$ & $\mathrm{Nd}$ & $\mathrm{v}$ \\
\hline & Putamen & Volume & $\mathrm{v}$ & $\mathrm{x}$ & $\mathrm{Nd}$ & $\mathrm{v}$ \\
\hline & Subcallosum gyrus & Volume & $\mathrm{x}$ & $\mathrm{v}$ & $\mathrm{Nd}$ & $\mathrm{v}$ \\
\hline & Mammillary body & Volume & $\mathrm{x}$ & $\mathrm{v}$ & $\mathrm{Nd}$ & $\mathrm{v}$ \\
\hline & Amygdala & Volume & $\mathrm{x}$ & $\mathrm{v}$ & $\mathrm{Nd}$ & $\mathrm{v}$ \\
\hline & Thalamus & Volume & $\mathrm{x}$ & $\mathrm{v}$ & $\mathrm{Nd}$ & $\mathrm{v}$ \\
\hline & Hypothalamus & Volume & $\mathrm{x}$ & $\mathrm{v}$ & $\mathrm{Nd}$ & $\mathrm{v}$ \\
\hline & Basal surface & Volume & $\mathrm{x}$ & $\mathrm{v}$ & $\mathrm{Nd}$ & $\mathrm{v}$ \\
\hline
\end{tabular}


Table 3 (continued)

\begin{tabular}{|c|c|c|c|c|c|c|}
\hline Reference & ROI & Parameters & Trans_vs_Natal Sex & Trans_vs_Opposite Sex & Trans_vs_Cis & M_vs_F \\
\hline \multirow[t]{18}{*}{ Manzouri et al. (2017) } & Cortex & $\mathrm{CTh}$ & $\mathrm{v}$ & $\mathrm{v}$ & $\mathrm{v}$ & $\mathrm{v}$ \\
\hline & Surface & SA & $\mathrm{x}$ & $\mathrm{v}$ & $\mathrm{Nd}$ & $\mathrm{v}$ \\
\hline & GM & Volume & $\mathrm{x}$ & $\mathrm{v}$ & $\mathrm{Nd}$ & $\mathrm{v}$ \\
\hline & Hippocampus R & Volume & $\mathrm{x}$ & $\mathrm{v}$ & $\mathrm{Nd}$ & $\mathrm{v}$ \\
\hline & Hippocampus L & Volume & $\mathrm{x}$ & $\mathrm{v}$ & $\mathrm{Nd}$ & $\mathrm{v}$ \\
\hline & Thalamus R & Volume & $\mathrm{x}$ & $\mathrm{v}$ & $\mathrm{Nd}$ & $\mathrm{v}$ \\
\hline & Thalamus L & Volume & $\mathrm{x}$ & $\mathrm{x}$ & $\mathrm{x}$ & $\mathrm{x}$ \\
\hline & Caudate R & Volume & $\mathrm{x}$ & $\mathrm{v}$ & $\mathrm{Nd}$ & $\mathrm{v}$ \\
\hline & Caudate L & Volume & $\mathrm{x}$ & $\mathrm{x}$ & $\mathrm{x}$ & $\mathrm{x}$ \\
\hline & Putamen R & Volume & $\mathrm{x}$ & $\mathrm{v}$ & $\mathrm{Nd}$ & $\mathrm{v}$ \\
\hline & Putamen L & Volume & $\mathrm{v}$ & $\mathrm{v}$ & $\mathrm{v}$ & $\mathrm{v}$ \\
\hline & Amygdala R & Volume & $\mathrm{x}$ & $\mathrm{x}$ & $\mathrm{x}$ & $\mathrm{x}$ \\
\hline & Amygdala L & Volume & $\mathrm{x}$ & $\mathrm{v}$ & $\mathrm{Nd}$ & $\mathrm{v}$ \\
\hline & Cerebellum R & Volume & $\mathrm{x}$ & $\mathrm{x}$ & $\mathrm{x}$ & $\mathrm{x}$ \\
\hline & Cerebellum L & Volume & $\mathrm{x}$ & $\mathrm{x}$ & $\mathrm{x}$ & $\mathrm{x}$ \\
\hline & Pallidum R & Volume & $\mathrm{x}$ & $\mathrm{x}$ & $\mathrm{x}$ & $\mathrm{x}$ \\
\hline & Pallidum L & Volume & $\mathrm{x}$ & $\mathrm{x}$ & $\mathrm{x}$ & $\mathrm{x}$ \\
\hline & Total intracranial & TIV & $\mathrm{x}$ & $\mathrm{v}$ & $\mathrm{Nd}$ & $\mathrm{v}$ \\
\hline \multirow[t]{7}{*}{ Pol et al. (2006) } & Intracranial & Volume & $\mathrm{x}$ & $\mathrm{v}$ & $\mathrm{x}$ & $\mathrm{v}$ \\
\hline & Total brain & Volume & $\mathrm{x}$ & $\mathrm{v}$ & $\mathrm{x}$ & $\mathrm{v}$ \\
\hline & Hypothalamus & Volume & $\mathrm{x}$ & $\mathrm{x}$ & $\mathrm{x}$ & $\mathrm{x}$ \\
\hline & 3rd ventricle & Volume & $\mathrm{x}$ & $\mathrm{x}$ & $\mathrm{x}$ & $\mathrm{x}$ \\
\hline & Lateral ventricle & Volume & $\mathrm{x}$ & $\mathrm{x}$ & $\mathrm{x}$ & $\mathrm{x}$ \\
\hline & GM & Volume & $\mathrm{x}$ & $\mathrm{x}$ & $\mathrm{x}$ & $\mathrm{x}$ \\
\hline & WM & Volume & $\mathrm{x}$ & $\mathrm{x}$ & $\mathrm{x}$ & $\mathrm{x}$ \\
\hline \multirow[t]{3}{*}{ Rametti et al. (2011a) } & SLF R & FA & $\mathrm{v}$ & $\mathrm{x}$ & $\mathrm{Nd}$ & $\mathrm{v}$ \\
\hline & Forceps minor & FA & $\mathrm{v}$ & $\mathrm{x}$ & $\mathrm{Nd}$ & $\mathrm{v}$ \\
\hline & CST & FA & $\mathrm{v}$ & $\mathrm{v}$ & $\mathrm{v}$ & $\mathrm{v}$ \\
\hline \multirow[t]{8}{*}{ Rametti et al. (2011b) } & GM & Volume & $\mathrm{x}$ & $\mathrm{v}$ & $\mathrm{Nd}$ & $\mathrm{v}$ \\
\hline & WM & Volume & $\mathrm{x}$ & $\mathrm{v}$ & $\mathrm{Nd}$ & $\mathrm{v}$ \\
\hline & CSF & Volume & $\mathrm{x}$ & $\mathrm{v}$ & $\mathrm{Nd}$ & $\mathrm{v}$ \\
\hline & Total intracranial & TIV & $\mathrm{x}$ & $\mathrm{v}$ & $\mathrm{Nd}$ & $\mathrm{v}$ \\
\hline & SLF R & FA & $\mathrm{v}$ & $\mathrm{v}$ & $\mathrm{v}$ & $\mathrm{v}$ \\
\hline & Forceps minor & FA & $\mathrm{v}$ & $\mathrm{v}$ & $\mathrm{v}$ & $\mathrm{v}$ \\
\hline & CST & FA & $\mathrm{v}$ & $\mathrm{v}$ & $\mathrm{v}$ & $\mathrm{v}$ \\
\hline & Anterior Cingulum R & FA & $\mathrm{v}$ & $\mathrm{v}$ & $\mathrm{v}$ & $\mathrm{v}$ \\
\hline \multirow[t]{8}{*}{ Savic and Arver (2011) } & Hippocampus & Volume & $\mathrm{x}$ & $\mathrm{Nd}$ & $\mathrm{Nd}$ & $\mathrm{v}$ \\
\hline & Thalamus & Volume & $\mathrm{v}$ & $\mathrm{v}$ & $\mathrm{v}$ & $\mathrm{x}$ \\
\hline & Caudate & Volume & $\mathrm{x}$ & $\mathrm{x}$ & $\mathrm{x}$ & $\mathrm{x}$ \\
\hline & Putamen & Volume & $\mathrm{v}$ & $\mathrm{v}$ & $\mathrm{v}$ & $\mathrm{x}$ \\
\hline & Total tissue & Volume & $\mathrm{x}$ & $\mathrm{Nd}$ & $\mathrm{Nd}$ & $\mathrm{v}$ \\
\hline & Total brain & Volume & $\mathrm{x}$ & $\mathrm{x}$ & $\mathrm{x}$ & $\mathrm{x}$ \\
\hline & GM & Volume & $\mathrm{x}$ & $\mathrm{x}$ & $\mathrm{x}$ & $\mathrm{x}$ \\
\hline & WM & Volume & $\mathrm{x}$ & $\mathrm{x}$ & $\mathrm{x}$ & $\mathrm{x}$ \\
\hline Yokota et al. (2005) & Corpus callosum & Shape & $\mathrm{v}$ & $\mathrm{v}$ & $\mathrm{v}$ & $\mathrm{v}$ \\
\hline
\end{tabular}


Table 3 (continued)

\begin{tabular}{|c|c|c|c|c|c|c|}
\hline Reference & ROI & Parameters & Trans_vs_Natal Sex & Trans_vs_Opposite Sex & Trans_vs_Cis & M_vs_F \\
\hline \multirow[t]{8}{*}{ Zubiaurre-Elorza et al. (2013) } & Cortex & CTh & $\mathrm{v}(\mathrm{MtF})-\mathrm{x}(\mathrm{FtM})$ & $\mathrm{x}(\mathrm{MtF})-\mathrm{v}(\mathrm{FtM})$ & $\mathrm{Nd}$ & $\mathrm{v}$ \\
\hline & Putamen R & Volume & $\mathrm{x}(\mathrm{MtF})-\mathrm{v}(\mathrm{FtM})$ & $\mathrm{x}(\mathrm{MtF})-\mathrm{x}(\mathrm{FtM})$ & $\mathrm{Nd}$ & $\mathrm{v}$ \\
\hline & Putamen L & Volume & $\mathrm{x}(\mathrm{MtF})-\mathrm{x}(\mathrm{FtM})$ & $\mathrm{x}(\mathrm{MtF})-\mathrm{x}(\mathrm{FtM})$ & $\mathrm{x}$ & $\mathrm{Nr}$ \\
\hline & Thalamus & Volume & $\mathrm{x}(\mathrm{MtF})-\mathrm{x}(\mathrm{FtM})$ & $\mathrm{x}(\mathrm{MtF})-\mathrm{x}(\mathrm{FtM})$ & $\mathrm{x}$ & $\mathrm{Nr}$ \\
\hline & Caudate & Volume & $\mathrm{x}(\mathrm{MtF})-\mathrm{x}(\mathrm{FtM})$ & $\mathrm{x}(\mathrm{MtF})-\mathrm{x}(\mathrm{FtM})$ & $\mathrm{x}$ & $\mathrm{Nr}$ \\
\hline & Pallidum & Volume & $\mathrm{x}(\mathrm{MtF})-\mathrm{x}(\mathrm{FtM})$ & $\mathrm{x}(\mathrm{MtF})-\mathrm{x}(\mathrm{FtM})$ & $\mathrm{x}$ & $\mathrm{Nr}$ \\
\hline & Hippocampus & Volume & $\mathrm{x}(\mathrm{MtF})-\mathrm{x}(\mathrm{FtM})$ & $\mathrm{x}(\mathrm{MtF})-\mathrm{x}(\mathrm{FtM})$ & $\mathrm{x}$ & $\mathrm{Nr}$ \\
\hline & Amygdala & Volume & $\mathrm{x}(\mathrm{MtF})-\mathrm{x}(\mathrm{FtM})$ & $\mathrm{x}(\mathrm{MtF})-\mathrm{x}(\mathrm{FtM})$ & $\mathrm{x}$ & $\mathrm{Nr}$ \\
\hline
\end{tabular}

Cis, natal and opposite sex; GM, gray matter; WM, white matter; CSF, cerebrospinal fluid; CST, corticospinal tract; SFG, superior frontal gyrus; SLF, superior longitudinal fasciculus; R, right; L, left; HCR, hemispheric connectivity ratio; LCW, lobar connectivity weight; MD, mean diffusivity; FA, fractional anisotropy; CTh, cortical thickness; SA, surface area; GMV, gray matter volume; TIV, total intracranial volume; v, differences; $\mathrm{x}$, non differences; $\mathrm{Nd}$, not definable; $\mathrm{Nr}$, not reported

FtM, $11 \mathrm{MC}$, and $12 \mathrm{FC}$ ) conducted with rs-fMRI investigated the resting state functional connectivity network and identified differences between cisgenders and transgenders in brain regions that seem to be involved in the neural network of body representation, and it concluded that different body representation may have different connectivity representation. However, it remains unclear whether or not a certain connectivity pattern is specific to transgenderism (Lin et al., 2014). A study (18 MtF, 13 FtM, 21 MC, 18 FC) conducted with rs-fMRI investigated functional connectivity patterns and reported that in pre-puberal children functional connectivity was similar in all groups (Nota et al., 2017), although stressed the necessity of increasing the sample to draw meaningful conclusions. Another study (24 MtF, $33 \mathrm{FtM}, 33 \mathrm{MC}$, $44 \mathrm{FC})$ that also investigated resting state functional connectivity (i.e., also a rs-fMRI study) found significant differences within a network around the supramarginal gyrus (i.e., a subregion within the parietal lobe) (Spies et al., 2016). A study ( $8 \mathrm{MtF}, 14 \mathrm{FtM}, 25 \mathrm{MC}, 26 \mathrm{FC}$ ) conducted with fMRI did not report any differences between transgender subjects and control group population during a verbal fluency test (Soleman et al., 2013). These four studies seem to suggest that in transgenders, brain activations have an intermediate pattern between those typical for their natal sex and experienced gender.

Four studies published in 2020 were additionally identified. An fMRI (emotional task) study on adolescents (mean age: 16.1 (FtM), $15.9(\mathrm{HeM}), 16.4(\mathrm{HeW})$ ) did not find differences in amygdala lateralization before hormonal (i.e., testosterone) treatment in FtM compared to $\mathrm{HeM}$ and $\mathrm{HeW}$ (Beking et al., 2020). A study comparing performance of 20 cisgender vs. 20 transgender individuals in gender face perception tasks showed bilateral activation differences in the precuneus of FtM compared to $\mathrm{FC}$. $\mathrm{MtF}$, in addition, significantly differed from $\mathrm{MC}$ in the lateral occipital cortex, posterior cingulate and angular gyri (Fisher et al., 2020). A study that conducted rs-MRI in young adults aged 19-22 years old, found MtF, FtM and FC having less resting state activations in parietal regions than $\mathrm{MC}$; and MtF having also weaker functional connectivity in some regions in frontal cortex than MC (Uribe et al., 2020). Similar to the study from Spies et al. (2016), also in rs-fMRI, this study suggests that in $\mathrm{MtF}$ some parietal and frontal cortex regions exhibit similar activation patterns as FC. Another fMRI study (body self-identification task) on 30 cisgenders and 30 transgenders found greater involvement of the limbic system in transgenders, who activated similar self- and body-processing neural systems aligned with their experienced gender and not with their birth-assigned sex (Majid et al., 2020).

\section{Sexual Orientation}

Eleven fMRI, 3 rs-fMRI, and 1 VBM studies published up to 2018 were analyzed. fMRI studies were conducted under visual stimulation (10) and an emotional judgment task (1). The 15 studies that conducted stereotaxic coordinates analysis involved $227 \mathrm{HoM}, 94 \mathrm{HoW}, 252 \mathrm{HeM}$, and $117 \mathrm{HeW}$. Figure 5 shows six representative slices with the foci resultant from the meta-analysis carried out using GingerAle 2.3.6 software (See Appendix 4 in Supplementary Materials for the labels of each foci and Table 6 for the number of foci related to different brain areas).

The meta-analysis "Homosexual_vs_Heterosexual Natal Sex" showed different activations in the visual area (BA 18 and 19). "Homosexual_vs_Heterosexual Opposite Sex" revealed differences in BA 23, which corresponds to the posterior cingular cortex, known to be involved in emotion, memory, meditation, and intrinsic control networks. Finally, the meta-analysis "Homosexual_vs_Heterosexual" showed 
Table 4 Regions of Interest analysis on sexual orientation

\begin{tabular}{|c|c|c|c|c|c|}
\hline Reference & ROI & Prameters & Homo_vs_Hetero Natal Sex & $\begin{array}{l}\text { Homo_vs_Hetero } \\
\text { Opposite Sex }\end{array}$ & M_vs_F \\
\hline \multirow[t]{13}{*}{ Abé et al. (2014) } & Middle temporal cortex L & CTh & $\mathrm{x}$ & $\mathrm{v}$ & $\mathrm{v}$ \\
\hline & Superior temporal cortex $\mathrm{L}$ & CTh & $\mathrm{x}$ & $\mathrm{x}$ & $\mathrm{v}$ \\
\hline & Inferior temporal cortex $\mathrm{R}$ & CTh & $\mathrm{v}$ & $\mathrm{v}$ & $\mathrm{v}$ \\
\hline & Lateral orbitofrontal cortex $\mathrm{R}$ & CTh & $\mathrm{v}$ & $\mathrm{x}$ & $\mathrm{v}$ \\
\hline & Pars Triangularis $\mathrm{R}$ & CTh & $\mathrm{v}$ & $\mathrm{v}$ & $\mathrm{v}$ \\
\hline & Lingual cortex $\mathrm{R}$ & CTh & $\mathrm{v}$ & $\mathrm{x}$ & $\mathrm{v}$ \\
\hline & Cuneus cortex $\mathrm{R}$ & CTh & $\mathrm{v}$ & $\mathrm{x}$ & $\mathrm{v}$ \\
\hline & Pericalcarine cortex $\mathrm{R}$ & CTh & $\mathrm{v}$ & $\mathrm{x}$ & $\mathrm{v}$ \\
\hline & Amygdala & Volume & $\mathrm{x}$ & $\mathrm{v}$ & $\mathrm{v}$ \\
\hline & Cerebellum & Volume & $\mathrm{x}$ & $\mathrm{v}$ & $\mathrm{v}$ \\
\hline & Hippocampus & Volume & $\mathrm{x}$ & $\mathrm{v}$ & $\mathrm{v}$ \\
\hline & Putamen & Volume & $\mathrm{x}$ & $\mathrm{v}$ & $\mathrm{v}$ \\
\hline & Thalamus & Volume & $\mathrm{v}$ & $\mathrm{v}$ & $\mathrm{v}$ \\
\hline \multirow[t]{8}{*}{ Manzouri and Savic (2018) } & Parietal lobe cortex & CTh & v (HoM)-x (HoW) & x (HoM)-v (HoW) & $\mathrm{v}$ \\
\hline & Superior temporal gyrus cortex & CTh & $\mathrm{x}(\mathrm{HoM})-\mathrm{x}(\mathrm{HoW})$ & v (HoM)-x (HoW) & $\mathrm{v}$ \\
\hline & Amygdala & Volume & x (HoM)-x (HoW) & v (HoM)-v (HoW) & $\mathrm{v}$ \\
\hline & Caudate & Volume & $\mathrm{x}(\mathrm{HoM})-\mathrm{x}(\mathrm{HoW})$ & $x(\mathrm{HoM})-\mathrm{x}(\mathrm{HoW})$ & $\mathrm{x}$ \\
\hline & Hippocampus & Volume & x (HoM)-x (HoW) & v (HoM)-v (HoW) & $\mathrm{v}$ \\
\hline & Putamen & Volume & $\mathrm{x}(\mathrm{HoM})-\mathrm{x}(\mathrm{HoW})$ & $\mathrm{x}(\mathrm{HoM})-\mathrm{x}(\mathrm{HoW})$ & $\mathrm{x}$ \\
\hline & Total intracranial & TIV & $\mathrm{x}(\mathrm{HoM})-\mathrm{x}(\mathrm{HoW})$ & $\mathrm{x}(\mathrm{HoM})-\mathrm{x}(\mathrm{HoW})$ & $\mathrm{x}$ \\
\hline & WM & FA & x (HoM)-x (HoW) & v (HoM)-v (HoW) & $\mathrm{v}$ \\
\hline \multirow[t]{4}{*}{ Savic and Lindström (2008) } & Cerebral hemisphere $\mathrm{R}$ & Volume & v (HoM)-v (HoW) & $\mathrm{x}(\mathrm{HoM})-\mathrm{x}(\mathrm{HoW})$ & $\mathrm{v}$ \\
\hline & Cerebral hemisphere L & Volume & x (HoM)-x (HoW) & $\mathrm{x}(\mathrm{HoM})-\mathrm{x}(\mathrm{HoW})$ & $\mathrm{x}$ \\
\hline & Cerebellar hemisphere $\mathrm{R}$ & Volume & x (HoM)-x (HoW) & x (HoM)-x (HoW) & $\mathrm{x}$ \\
\hline & Cerebellar hemisphere L & Volume & $\mathrm{x}(\mathrm{HoM})-\mathrm{x}(\mathrm{HoW})$ & $\mathrm{x}(\mathrm{HoM})-\mathrm{x}(\mathrm{HoW})$ & $\mathrm{x}$ \\
\hline \multirow[t]{6}{*}{ Witelson et al. (2008) } & Anterior half CC & Volume & $\mathrm{x}$ & $\mathrm{Nr}$ & $\mathrm{Nr}$ \\
\hline & Posterior mid-body CC & Volume & $\mathrm{x}$ & $\mathrm{Nr}$ & $\mathrm{Nr}$ \\
\hline & Isthmus CC & Volume & $\mathrm{v}$ & $\mathrm{Nr}$ & $\mathrm{Nr}$ \\
\hline & Mid-sagittal area CC & Volume & $\mathrm{x}$ & $\mathrm{Nr}$ & $\mathrm{Nr}$ \\
\hline & Splenium CC & Volume & $\mathrm{x}$ & $\mathrm{Nr}$ & $\mathrm{Nr}$ \\
\hline & Total CC & Volume & $\mathrm{x}$ & $\mathrm{Nr}$ & $\mathrm{Nr}$ \\
\hline
\end{tabular}

WM, white matter; CC, corpus callosum; CTh, cortical thickness; TIV, total intracranial volume; FA, fractional anisotropy; $\mathrm{v}$, differences; $\mathrm{x}$, non differences; $\mathrm{Nr}$, Not reported

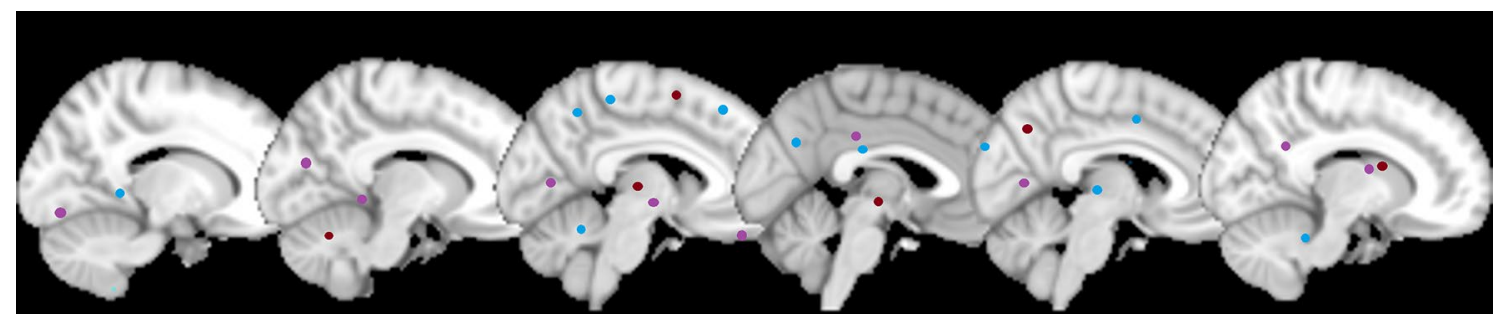

Fig. 4 Six representative sagittal slices showing the foci resultant from the meta-analyses of stereotaxic coordinates where significant differences were found between groups with opposite gender identity (result from GingerAle 2.3.6; figure generated with micron.exe) (Purple $=$ Transgender_vs_Natal sex; red $=$ Transgender_vs_Opposite sex; blue $=$ Transgender_vs_Natal and Opposite Sex) 
Table 5 Stereotaxic coordinates analysis on gender identity (number of foci related to different brain areas)

\begin{tabular}{|c|c|c|c|c|c|}
\hline \multicolumn{2}{|c|}{$\begin{array}{l}\text { Transgender_vs_Cisgender Natal } \\
\text { Sex }\end{array}$} & \multicolumn{2}{|c|}{$\begin{array}{l}\text { Transgender_vs_Cisgender } \\
\text { Opposite Sex }\end{array}$} & \multicolumn{2}{|l|}{ Transgender_vs_Cisgender } \\
\hline BA 18 & 7 & BA 19 & 4 & BA 18 & 9 \\
\hline Thalamus & 2 & Crebellum & 2 & BA 10 & 6 \\
\hline BA 10 & 2 & BA 9 & 2 & BA 19 & 6 \\
\hline BA 22 & 2 & Insula & 2 & Insula & 4 \\
\hline BA 23 & 2 & Anterior cingulate & 1 & BA 32 & 4 \\
\hline Insula & 1 & Frontal gyrus & 1 & Thalamus & 3 \\
\hline Caudate & 1 & Posterior cingulate & 1 & BA 22 & 3 \\
\hline Gyrus precuneus & 1 & Putamen & 1 & Brainstem & 2 \\
\hline Hypothalamus & 1 & Thalamus & 1 & Cerebellum & 2 \\
\hline Midbrain & 1 & BA 10 & 1 & Frontal gyrus & 2 \\
\hline Parahippocampal gyrus & 1 & BA 22 & 1 & Hypothalamus & 2 \\
\hline Perisylvian & 1 & BA 24 & 1 & Posterior cingulate cortex & 2 \\
\hline Substantia & 1 & BA 39 & 1 & BA 9 & 2 \\
\hline BA 6 & 1 & & & BA 31 & 2 \\
\hline BA 9 & 1 & & & BA 23 & 1 \\
\hline BA 11 & 4 & & & Anterior cingulate cortex & 1 \\
\hline BA 17 & 3 & & & Caudate & 1 \\
\hline BA 19 & 2 & & & Fusiform & 1 \\
\hline BA 31 & 5 & & & Hippocampus & 1 \\
\hline \multirow[t]{12}{*}{ BA 32} & 1 & & & Perisylvian & 1 \\
\hline & & & & Precentral gyrus & 1 \\
\hline & & & & Putamen & 1 \\
\hline & & & & Temporal gyrus & 1 \\
\hline & & & & BA 4 & 1 \\
\hline & & & & BA 5 & 1 \\
\hline & & & & BA 7 & 1 \\
\hline & & & & BA 8 & 1 \\
\hline & & & & BA 21 & 1 \\
\hline & & & & BA 24 & 1 \\
\hline & & & & BA 37 & 1 \\
\hline & & & & BA 40 & 1 \\
\hline
\end{tabular}

BA, Brodmann area; Transgender_vs_Cisgender Natal Sex, MtF_vs_MC+FtM_vs_FC; Transgender_vs_ Cisgender Opposite Sex, MtF_vs_FC+FtM_vs_MC

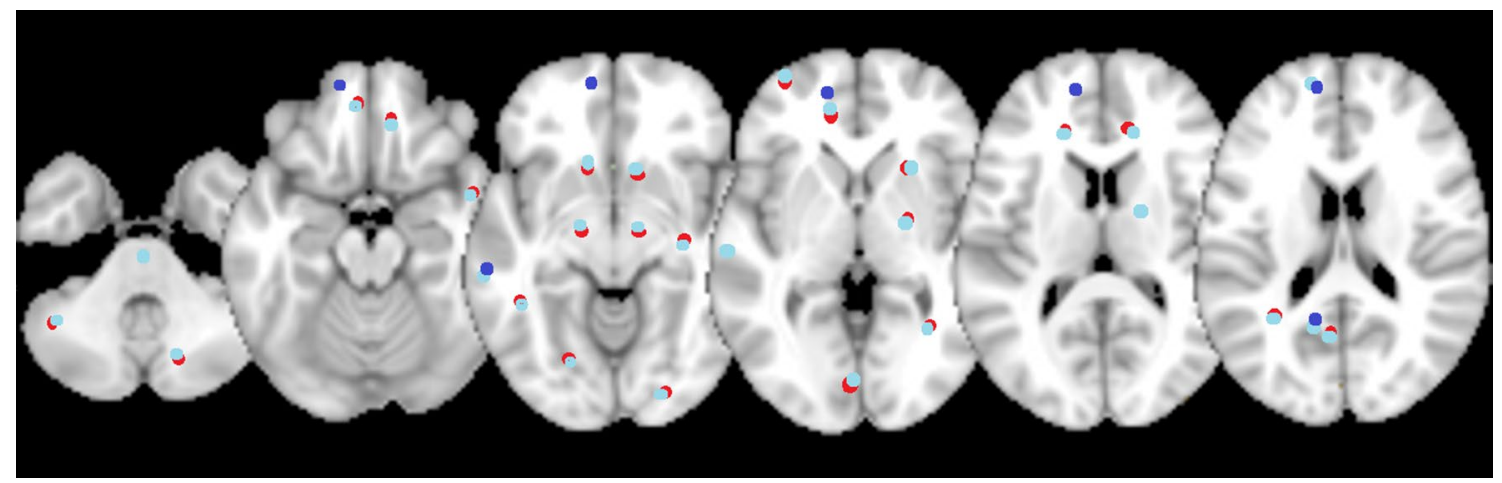

Fig. 5 Six representative axial slices showing the foci resultant from the meta-analyses of stereotaxic coordinates where significant differences were found between groups with opposite sexual orientation (result from GingerAle 2.3.6; figure generated with micron.exe)
(Red=Homosexual_vs_Heterosexual Natal Sex; cyan=Homosexual_vs_Heterosexual Opposite Sex; blue=Transgender_vs_Natal and Opposite Sex; indigo =Homosexual_vs_Heterosexual) 
Table 6 Stereotaxic coordinates analysis on sexual orientation (number of foci related to different brain areas)

\begin{tabular}{|c|c|c|c|c|c|}
\hline \multicolumn{2}{|c|}{ Homosexual_vs_Heterosexual Natal Sex } & \multicolumn{2}{|c|}{$\begin{array}{l}\text { Homosexual_vs_Heterosexual } \\
\text { Opposite Sex }\end{array}$} & \multicolumn{2}{|c|}{$\begin{array}{l}\text { Homosexual_vs_Hetero- } \\
\text { sexual }\end{array}$} \\
\hline BA 19 & 10 & BA 23 & 3 & BA 18 & 5 \\
\hline BA 11 & 7 & Coroide plexus & 1 & BA 23 & 4 \\
\hline BA 18 & 6 & BA 17 & 1 & Caudate & 3 \\
\hline Caudate & 5 & BA 21 & 1 & BA 17 & 3 \\
\hline Cingulate & 5 & BA 25 & 1 & BA 19 & 3 \\
\hline Cerebellum & 4 & BA 41 & 1 & BA 40 & 3 \\
\hline BA 7 & 4 & BA 45 & 1 & Cingulate & 2 \\
\hline Optical radiation & 3 & & & Insula & 2 \\
\hline BA 10 & 3 & & & Thalamus & 2 \\
\hline BA 17 & 3 & & & BA 10 & 2 \\
\hline BA 40 & 3 & & & BA 11 & 2 \\
\hline Amygdala & 2 & & & BA 24 & 2 \\
\hline Brain stem & 2 & & & BA 39 & 2 \\
\hline Insula & 2 & & & Brain stem & 1 \\
\hline Striatum & 2 & & & Coroide plexus & 1 \\
\hline Thalamus & 2 & & & Globus pallidus & 1 \\
\hline BA 22 & 2 & & & Lateral sulcus & 1 \\
\hline BA 24 & 2 & & & Optical radiation & 1 \\
\hline BA 32 & 2 & & & Periaqueductal & 1 \\
\hline BA 39 & 2 & & & Posterior cingulate & 1 \\
\hline Anterior cingulate cortex & 1 & & & Putamen & 1 \\
\hline Corpus callosum & 1 & & & Singular gyrus & 1 \\
\hline Globus pallidus & 1 & & & Striatum & 1 \\
\hline Hippocampus & 1 & & & BA 4 & 1 \\
\hline Paracentral lobule & 1 & & & BA 7 & 1 \\
\hline Periaqueductal & 1 & & & BA 21 & 1 \\
\hline Postcentral gyrus & 1 & & & BA 22 & 1 \\
\hline Posterior cingulate & 1 & & & BA 25 & 1 \\
\hline Precentral gyrus & 1 & & & BA 31 & 1 \\
\hline Putamen & 1 & & & BA 32 & 1 \\
\hline Temporal pole & 1 & & & BA 41 & 1 \\
\hline Temporo parietal junction & 1 & & & BA 45 & 1 \\
\hline BA 6 & 1 & & & BA 47 & 1 \\
\hline BA 23 & 1 & & & & \\
\hline BA 25 & 1 & & & & \\
\hline BA 30 & 1 & & & & \\
\hline BA 31 & 1 & & & & \\
\hline
\end{tabular}

BA, Brodmann area; Homosexual_vs_Heterosexual Natal Sex, HoM_vs_HeM+HoW_vs_HeW; Homosexual_vs_Heterosexual Opposite Sex, HoM_vs_HeW + HoW_vs_HeM that homosexual people's brain activation differs more frequently in all these three areas.

From April 2018 to March 2021, two additional publications exploring sexual orientation using fMRI were identified. One study (Folkierska-Żukowska et al., 2020) considered within-group variations attributable to nonconforming behaviors and applied a mental rotation task to 90 individuals (mean age: 27.09 (23 HoM nonconforming), 26.6 (23 HoM conforming), 25.59 (22 HeM), 26.33 (23 HeW) yearls old). The study recalls that the term nonconforming refers to sex-atypical behaviors, interests, hobbies, activity levels, and play partner preferences, which have been "reliably associated with human nonheterosexuality" (FolkierskaŻukowska et al., 2020). This study found similarities in activation patterns from nonconforming $\mathrm{HoM}$ and $\mathrm{HeW}$ in right superior frontal gyrus, tight angular gyrus, right amygdala, parahippocampal gyrus, middle temporal gyrus and precuneus, which authors referred as "cross-sex shift". 
Both conforming and nonconforming HoM had statistically significantly different levels of activations from $\mathrm{HeW}$ in left medial superior frontal gyrus and in the precentral/ paracentral gyri. Patterns of activations in the angular and middle temporal gyri differed between conforming and non-conforming HoM highlighting heterogeneity in brain function within HoM. The other study (Afdile et al., 2019) applied a social grouping task involving $14 \mathrm{HoM}$ and 15 $\mathrm{HeM}$, and found significant group differences in areas of the medial prefrontal cortex, frontal pole, anterior cingulate cortex, right temporal parietal junction and bilateral superior frontal gyri.

\section{Metabolic Analysis}

\section{Gender Identity}

Three PET and one SPECT studies were analyzed. They involved $25 \mathrm{FtM}, 45 \mathrm{MtF}, 41 \mathrm{FC}$, and $49 \mathrm{MC}$. A PET study investigated the hypothalamic network in 12 gynephilic (i.e., sexual preference for women) MtF, 12 gynephilic MC, and 12 androphilic (i.e., sexual preference for men) FC under smelling stimulation with steroids. Transgender individuals reported an intermediate hypothalamic pattern of activation between males and females, with prevalent feminine features (Berglund et al., 2008). Another PET study investigated serotonin transporter distribution in 14 $\mathrm{MtF}$ of different sexual orientations, $13 \mathrm{MC}$, and $9 \mathrm{FC}$ with unspecified sexual orientation. While MC reported a rightward asymmetry in the midcingulate cortex, $\mathrm{MtF}$ and FC did not (Kranz et al., 2014a). Another PET study investigated serotonin transporter distribution in $19 \mathrm{MtF}$, $14 \mathrm{FtM}, 24 \mathrm{MC}$, and $11 \mathrm{FC}$. ROIs investigated included insular cortex, cingulate cortex, amygdala, caudate, hippocampus, hypothalamus, putamen, and thalamus. Sexual orientation was not specified for any of them. Serotonin reuptake transporter non-displaceable binding potential (BPnd) was lower in amygdala, caudate, insular cortex, hippocampus, and putamen in FtM with respect to MC (Kranz et al., 2015). One SPECT study investigated regional cerebral blood flow (rCBF) in 11 gynephilic FtM and 9 androphilic FC. Transgender subjects reported an increase in $\mathrm{rCBF}$ in the right insula and a decrease in rCBF in the left anterior cingulate cortex (ACC) (Nawata et al., 2010). Taken together, these results seem to suggest that in the studies analyzed, transgender people not under hormonal treatment have certain brain metabolic features which tend to be slightly different from their natal sex and which are either similar to the opposite sex or intermediate between the two sexes.

\section{Sexual Orientation}

Four PET studies were analyzed. They involved $32 \mathrm{HoM}, 24$ $\mathrm{HoW}, 44 \mathrm{HeM}$, and $37 \mathrm{HeW}$. Hypothalamic activation under smelling stimulation with AND (i.e., progesterone derivative) and EST (i.e., estrogen-like steroid) was investigated in two studies (Berglund et al., 2006; Savic et al., 2005). Berglund et al. found a different preoptic hypothalamus activation between HoW and HeM with AND, while Savic et al. found a different preoptic and ventromedial hypothalamus activation between HoW and HeM with AND. Another study explored the brain activation in $8 \mathrm{HoM}$ and $7 \mathrm{HeM}$ in response to fluoxetine (i.e., selective serotonin reuptake inhibitor). With regards to the areas which are known to play a role in sexual behavior, HoM were reported to have a lower decrease in hypothalamic glucose metabolism than HeM. With regard to the areas which are not known to play a role in sexual behavior, HoM exhibited an increase of the glucose metabolism in the cingulate cortex, where HeM were reported to have a decrease; and in the prefrontal cortex, where no changes were reported for HeM (Kinnunen et al., 2004). Another study analyzed functional connectivity in $12 \mathrm{HoM}, 12 \mathrm{HoW}, 13 \mathrm{HeM}$, and $13 \mathrm{HeW}$. HoM and HeW exhibited more connections from the left amygdala with the contralateral amygdala, hypothalamus, subcallosum, and the anterior cingulate, while HoW and HeM exhibited more connections from the right amygdala with caudate and putamen (Savic \& Lindström, 2008). Taken together, these results seem to suggest that homosexual individuals have some brain metabolic features that slightly differ from heterosexual individuals of their natal sex and others that are similar to heterosexuals of the opposite sex.

\section{Analysis of Bias}

Appendix 5 and 6 show the risk of bias of the papers published up to 2018, calculated using the Quadas tool (see Supplementary Materials). Only 5/14 questions were applicable to our research. In all studies on both gender identity and sexual orientation, the samples were not representative of the population. Selection criteria were described clearly in only $31 / 51$ papers (i.e., $16 / 30$ on gender identity and $15 / 21$ on sexual orientation). Texts were explanatory enough so as it can be replicated in 44/51 papers (i.e., 24/30 on gender identity and $20 / 21$ on sexual orientation). Intermediate results were reported in $46 / 51$ papers (i.e., $26 / 30$ on gender identity and $20 / 21$ on sexual orientation). Withdrawals (i.e., individuals who enrol a study and withdraw from it afterwards) from the studies included in this review were explained in all cases that referred it (i.e., $5 / 5$ studies on gender identity and $3 / 3$ studies on sexual orientation). 


\section{Discussion}

\section{Main Findings}

The results from our systematic review and meta-analyses do not allow us to conclude on the specific brain phenotypes differential for each of the groups covered by this review. Although functional MRI studies (i.e., involving either fMRI or rs-MRI) on gender identity seem to indicate that fronto-parietal and cingulo-opercular brain regions are differentially relevant in transgenderism, a clear pattern accompanied by consistent structural changes is still to be found. Studies on gender identity with moderate-to-larger samples which included individuals with different sexual orientation in their control groups (Baldinger-Melich et al., 2020; Manzouri \& Savic, 2019), exposed the complexities underlying both gender identity and sexual orientation. The data extracted may suggest that before hormonal treatment the majority of transgenders' brain features covered by the studies reviewed could be similar to those of their natal sex, but certainly some brain parameters differ resembling those of their experienced gender. Also, although homosexual's neuroanatomy, neurophysiology, and neurometabolism may tend to resemble those of heterosexual individuals of their same sex, some brain features differ and are similar to those of heterosexual individuals of the opposite sex in some of the studies analyzed.

The compilation of the data from the studies included shows neural differences between the groups studied. However, brain functions are mediated by different brain areas and their interactions, rather than by single structures. The correlation or association between a certain brain function, volumetric change or activation, with a certain activity and/ or behavior does not establish whether (or not) that structure/function is causally important for that activity/behavior (Koob et al., 2013; Maney, 2016). It merely shows a possible involvement or apparent trend. Complex human behaviors (and few simple behaviors) cannot be entirely explained by phenomena occurring only in a single brain region. Therefore, the idea that brain sexual differences cause behavioral sexual differences, rather than being an assumption, still constitutes a hypothesis to verify.

Studies on cisgender and heterosexual samples have reported sex differences in brain anatomy on a global scale, regarding absolute volumes (Kurth et al., 2016). Studies have also reported sexual dimorphism in the relative sizes and shapes of regional brain structures, with the direction of the sex effect varying between regions, including the Broca's region (Kurth et al., 2016), corpus callosum (Prendergast et al., 2015), amygdala and hippocampus (Giedd et al., 1996). These findings reflect on the selectivity of the brain regions analyzed by the studies included in this review. However, research investigating differences at the level of regional tissue volumes is highly contradictory. A large study that analyzed MRI data of 1400 cisgender heterosexual individuals from four different datasets (Joel et al., 2015) found substantial overlap in the distribution of anatomical traits between males and females in all brain regions and connections examined, undermining attempts to clearly distinguish between "male" and "female" forms of specific brain features. They arrived at the idea that human brains cannot in fact, be distinctly categorized into two distinct classes but rather, that male and female brains are comprised of "unique mosaics" of features, some of which are more common in one sex than the other and some that are common in both.

Some authors refer to an early programming of gender and sexual inclination driven by sexual differentiation in the brain, proposing that the latter influences the development of the brain areas modulating body perception (i.e., related to gender identity) or sexual arousal (i.e., related to sexual orientation) (Burke et al., 2017; Manzouri \& Savic, 2019). Others underline the interaction between brain, culture and behavior, arguing that structural and functional brain changes in transgender individuals may be consequence of culture and behavior (Mohammadi \& Khalegi, 2018). The etiology and drivers of differences in gender identity and sexual orientation is out of the scope of this review, and caution must be exercised to drive conclusions from the neuroscience literature alone, as human behavior, ultimately, is not reducible to biological nor to cultural factors, but is a consequence of their interaction. As such, human sexuality is a multilevel complex, and the challenge is to investigate how biological, historical and cultural elements interact with each other.

\section{Regions of Interest Analysis}

The lack of data did not allow us to meta-analyze the information obtained from the studies that conducted ROI analyses. From extracting and summarizing all the information available, differences were found between cisgender and transgender people in white matter microstructure, volumetric analyses, cortical thickness, and corpus callosum shape. Differences between heterosexual and homosexual people were found in cortical thickness, subcortical volumes, and cerebral hemisphere, but not in white matter tracts. The studies included, in the rest of the ROIs analyzed, either did not find significant differences between cisgender and transgender brains nor between heterosexual and homosexual; or found significant differences just between transgenders and opposite sex cisgenders, and between homosexuals and opposite sex heterosexuals (see Tables 3 and 4). Our findings on gender identity are consistent with previous studies that also attempted to summarize the literature findings on this topic, according to which gross morphology in transgenders is more similar to cisgender people of their natal sex than to cisgender 
people of their experienced gender (Guillamon et al., 2016; Kreukels \& Guillamon, 2016; Mueller et al., 2017; Smith et al., 2015), even though white matter microstructure (Kreukels \& Guillamon, 2016; Mueller et al., 2017; Smith et al., 2015), cortical thickness (Guillamon et al., 2016; Smith et al., 2015), and subcortical volumes (Mueller et al., 2017) may deviate from the biological sex towards values of experienced gender.

\section{Stereotaxic Coordinates Analysis}

Occipital brain regions, involved in visual processing, are the ones that most frequently were found to have a different activation in cisgenders compared to transgenders, followed by some fronto-temporal foci. This is not surprising given that, in general, most fMRI studies involved in both analyses involved visual stimulation. In addition, specifically the BA 23 had different activations for heterosexuals with respect to homosexuals. Our meta-analysis found different brain activations between different groups scattered across the whole brain, but overall with low frequency (see Tables 5 and 6). Our results on gender identity are consistent with some of the previous studies mentioned above, according to which in certain brain areas transgenders' activation is closer to those of their experienced gender (Guillamon et al., 2016; Smith et al., 2015). While there is still concensus that a clear picture has yet to emerge (Mueller et al., 2017), recent advances in artificial intelligence confirm the observations above, by indicating that some fronto-parietal and cingulo-opercular areas may be of relevance for predicting hormonal therapy outcomes (Moody et al., 2021).

\section{Metabolic Analysis}

In transgenders and homosexuals, some metabolic features seem to differ slightly from cisgenders of their natal sex and from heterosexuals of their natal sex respectively. However, given the reduced number of studies included that conducted these analyses, these findings cannot be generalized. This is in line with what the scientific literature on gender identity up to date has concluded in this respect (Smith et al., 2015).

\section{Strengths and Limitations}

To the best of our knowledge, this is the first systematic review and meta-analysis of the neuroimaging literature on structural, functional, and metabolic differences as a function of both gender identity (before the hormonal treatment) and sexual orientation. In addition, we carefully extracted and processed all data from all studies considered for metaanalyses and made them publicly available to facilitate further research in this important area.
Several limitations regarding the small sample size of the meta-analysis and the heterogeneity of the investigations must be acknowledged. The analyses of our systematic search up to 2018 included 51 studies (i.e., 30 on gender identity and 21 on sexual orientation) all with relatively small samples, conducted with different neuroimaging techniques (1 SPECT, 3 PET, 6 fMRI, 8 rs-fMRI, and 13 MRI on gender identity; 4 PET, 5 MRI, 3 rs-fMRI, and $11 \mathrm{fMRI}$ on sexual orientation). Different studies conducted with MRI investigated different brain structures (cortex, subcortical volumes, white matter, CSF, and ventricles in gender identity; cortex, subcortical volumes, and white matter in sexual orientation). fMRI was conducted under different stimulations ( 1 smelling, 1 vocal stimulation, 1 mental rotation task, 1 verbal fluency test, and 2 visual in gender identity; 10 visual stimulation and 1 emotional judgment task in sexual orientation). Metabolic analysis investigated different brain areas (hypothalamic network, serotonin transport distribution in different ROI, and rCBF in gender identity studies; hypothalamic activation and functional connectivity in sexual orientation studies) using different neuroimaging techniques (PET and SPECT in gender identity research; PET in sexual orientation research). As a result, it was not possible to meta-analyze the results from all studies that fit our inclusion/exclusion criteria, and the main contribution of our work, therefore, is limited to the scientific compilation and synthesis of the data available. An update on the primary search conducted in one database, added 12 papers to the analyses which, although enriched the data presented, was rather confirmatory of our main findings and added heterogeneity to the results.

Moreover, some studies had some limitations regarding the presentation of their data. First, some studies did not report statistical parameters and just reported whether or not there were significant differences between cisgenders and transgenders and between heterosexuals and homosexuals. Second, other studies reported statistical parameters only in case of significant differences between groups, and omitted reporting negative results (i.e., when no differences were found) (gender identity investigation: Burke et al., 2014; Kranz et al., 2014b, 2015; Ku et al., 2013; Lin et al., 2014; Luders et al., 2009; Nota et al., 2017; Pol et al., 2006; Santarnecchi et al., 2012; Soleman et al., 2013; Spies et al., 2016; Yokota et al., 2005; Zubiaurre-Elorza et al., 2013; sexual orientation investigation: Hu et al., 2008; Ponseti et al., 2007; Savic and Lindström, 2008; Sylva, 2013; Zeki \& Romaya, 2010; for more detailed information, please see analysis of bias in Appendix 5 and 6). A complete presentation of scientific data, including negative results, is important to precisely evaluate scientific investigations on a certain topic (Matosin et al., 2014).

Information on the biological sex of the studies' participants is part of the scientific data we collected and made available. The data presented show $\mathrm{MtF}$ and FtM transgender 
individuals do not have mirror images of brain differences. However, the heterogeneity of the design of the studies involved, despite enriching the scope of this review, due to the limited number of studies included and their sample sizes, made it impossible to draw conclusions on specific biological sex differences for the groups covered in this review. For example, some papers compared $\mathrm{MtF}$ with $\mathrm{MC}$, others $\mathrm{MtF}$ with FC, others FtM with MC, and others FtM with FC. The studies included in this review on transgenderism did not provide information on early-onset or late-onset transgenderism. Therefore, analysis and information of this important point is lacking.

Finally, as Guillamon et al. (2016) noted, some studies conducted on gender identity did not report the sexual orientation of the individuals that constituted their sample. Gender identity and sexual orientation are conceptually different, i.e., both cisgender and transgender people are either heterosexual or homosexual (Burke et al., 2017; Moser, 2010), and there are more gender identities other than cis-/transgender(ism) (such as genderqueer or non-binary) and other sexual orientations other than hetero-/homosexual(ism) (such as bi-, pan-, and asexual). Sexual orientation could be associated with brain structural specific features regardless and independently from gender identity as some recent studies suggest (Baldinger-Melich et al., 2020; Manzouri \& Savic, 2019). Thus, meaning that the structural, functional, and metabolic variations found in homosexual transgenders with respect to heterosexual cisgenders may be related to their sexual orientation rather than to their gender identity (Blanchard et al., 1987). A recent study identified brain regions where both sexual orientation and gender identity seemingly interact (Wang, Han, et al., 2020).

\section{Conclusions and Future Directions}

Over the past few years, the neuroimaging investigation on human sexuality has increased and several studies on gender identity and sexual orientation comparing cisgenders vs. transgenders and heterosexuals vs. homosexuals have been conducted.

This review explored structural, functional, and metabolic features of cisgenders compared to transgenders before hormonal treatment and heterosexuals compared with homosexuals. Results suggest that, although the majority of neuroanatomical, neurophysiological, and neurometabolic features in transgenders resemble those of their natal sex rather than those of their experienced gender, and in homosexuals these resemble those of their same-sex heterosexual population rather than their opposite sex heterosexual population, in the gender identity investigation, in $\mathrm{MtF}$ it was possible to find traits which are "feminine and demasculinized" and in FtM it was possible to find traits which are "masculine and defeminized" (Kreukels \& Guillamon, 2016). The same could be said with regard to the investigation on sexual orientation, where some brain features in the homosexual population from the studies reviewed resembled those of the heterosexual population of their opposite sex. Due to conflicting results, it was, however, not possible to identify specific brain features which consistently differ between cisgender and transgender nor between heterosexual and homosexual groups. Very small brain changes, to date undetectable using the current neuroimaging tools, may affect behavior. The small number of studies, the small sample size of each study, the heterogeneity of investigations, the lack of negative results reported by some studies, and the fact that some studies did not report the sexual orientation of the individuals that composed their sample did not allow drawing general conclusions. Moreover, as the samples of the publications involved are not representative of the population analyzed, caution should be taken in the interpretation of the results of this review.

To overcome the limitations mentioned above, future studies should: (1) keep investigating brain areas which are sexually dimorphic (e.g., hypothalamus, hippocampus, caudate, corpus callosum, and serotonin transport) and brain areas involved in processing own-body perception (e.g., parietal, frontal, insular cortex, and its connections with thalamus and putamen) and sexual stimuli and arousal (e.g., hypothalamus and ventral striatum); (2) conduct Metabolic Analysis along with structural and functional to increase the number of data available; (3) report both positive and negative results to conduct an unbiased statistical analysis; (4) report sexual orientation of individuals that comprise the sample size in studies on gender identity; (5) increase the sample size and expand the age range of the sample; (6) differentiate with respect to early- or late-onset transgenderism to reach a better understanding of the biological features underlying them. Future reviews in the topic should extend the inclusion criteria to distinguish between pre- vs. post-pubertal and pre- vs. post-hormonal treatment, as well as include other advanced neuroimaging modalities such as magnetic resonance spectroscopy, and dynamic sequence acquisitions to increase the value and scope of the present report.

Acknowledgements LB was partially funded by Science and Engineering Research Council (Grant No. EP/M005976/1).

\section{Declarations}

Conflict of interest We declare no competing interests.

Open Access This article is licensed under a Creative Commons Attribution 4.0 International License, which permits use, sharing, adaptation, distribution and reproduction in any medium or format, as long as you give appropriate credit to the original author(s) and the source, provide a link to the Creative Commons licence, and indicate if changes were made. The images or other third party material in this article are included in the article's Creative Commons licence, unless indicated otherwise in a credit line to the material. If material is not included in 
the article's Creative Commons licence and your intended use is not permitted by statutory regulation or exceeds the permitted use, you will need to obtain permission directly from the copyright holder. To view a copy of this licence, visit http://creativecommons.org/licenses/by/4.0/.

\section{References}

Abé, C., Johansson, E., Allzén, E., \& Savic, I. (2014). Sexual orientation related differences in cortical thickness in male individuals. PLoS ONE, 9(12), e114721. https://doi.org/10.1371/journal.pone. 0114721

Afdile, M., Jääskeläinen, I. P., Glerean, E., Smirnov, D., Alho, J., Äimälä, A., \& Sams, M. (2019). Contextual knowledge provided by a movie biases implicit perception of the protagonist. Social Cognitive and Affective Neuroscience, 14(5), 519-527. https:// doi.org/10.1093/scan/nsz028

Allen, L. S., \& Gorski, R. A. (1992). Sexual orientation and the size of the anterior commissure in the human brain. Proceedings of the National Academy of Sciences, USA, 89(15), 7199-7202. https:// doi.org/10.1073/pnas.89.15.7199

Altinay, M., \& Anand, A. (2020). Neuroimaging gender dysphoria: a novel psychobiological model. Brain Imaging and Behavior, 14(4), 1281-1297. https://doi.org/10.1007/s11682-019-00121-8

American Psychological Association. (2008). Answers to your questions for a better understanding of sexual orientation \& homosexuality. Retrieved from September 13, 2018, http://www.apa.org/topics/ lgbt/orientation.aspx

American Psychiatric Association. (2013). Diagnostic and statistical manual of mental disorders (5th edn.). American Psychiatric Publishing.

American Psychological Association. (2014). Answers to your questions about transgender people, gender identity \& gender expression. Retrieved from September 13, 2018, http://www.apa.org/topics/ lgbt/transgender.aspx

Baldinger-Melich, P., Urquijo Castro, M. F., Seiger, R., Ruef, A., Dwyer, D. B., Kranz, G. S., Klöbl, M., Kambeitz, J., Kaufmann, U., Windischberger, C., Kasper, S., Falkai, P., Lanzenberger, R., \& Koutsouleris, N. (2020). Sex matters: A multivariate pattern analysis of sex- and gender-related neuroanatomical differences in cisand transgender individuals using structural magnetic resonance imaging. Cerebral Cortex, 30(3), 1345-1356. https://doi.org/10. 1093/cercor/bhz170

Balthazar, J. (2016). Sex differences in partner preferences in humans and animals. Philosophical Transactions of the Royal Society of London Series B, Biological Sciences, 371(1688), 20150118

Beking, T., Burke, S. M., Geuze, R. H., Staphorsius, A. S., Bakker, J., Groothuis, A. G. G., \& Kreukels, B. P. C. (2020). Testosterone effects on functional amygdala lateralization: A study in adolescent transgender boys and cisgender boys and girls. Psychoneuroendocrinology, 111, 104461. https://doi.org/10.1016/j.psyne uen.2019.104461

Berglund, H. H., Lindström, P., Dhejne-Helmy, C., \& Savic, I. (2008). Male-to-female transsexuals show sex-atypical hypothalamic activation when smelling odorous steroids. Cerebral Cortex, 18(8), 1900-1908. https://doi.org/10.1093/cercor/bhm216

Berglund, H., Lindström, P., \& Savic, I. (2006). Brain response to putative pheromones in lesbian women. Proceedings of the National Academy of Sciences, USA, 103(21), 8269-8274. https://doi.org/ 10.1073/pnas.0600331103

Blanchard, R., Clemmensen, L. H., \& Steiner, B. W. (1987). Heterosexual and homosexual gender dysphoria. Archives of Sexual Behavior, 16(2), 139-152. https://doi.org/10.1007/BF01542067

Burke, S. M., Cohen-Kettenis, P. T., Veltman, D. J., Klink, D. T., \& Bakker, J. (2014). Hypothalamic response to the chemo-signal androstadienone in gender dysphoric children and adolescents. Frontier in Neuroendocrinology, 5, 1-10. https://doi.org/10.3389/ fendo.2014.00060

Burke, S. M., Kreukels, B. P., Cohen-KEttenis, P. T., Veltman, D. J., Klink, D. T., \& Bakker, J. (2016). Male-typical visuospatial functioning in gynephilic girls with gender dysphoria. Organizational and activational effects of testosterone. Journal of Psychiatry and Neuroscience, 41(6), 395-404. https://doi.org/10.1503/jpn. 150147

Burke, S. M., Manzouri, A. H., \& Savic, I. (2017). Structural connections in the brain in relation to gender identity and sexual orientation. Scientific Reports, 7(1), 17954-17965. https://doi.org/10. 1038/s41598-017-17352-8

Butler, J. (1990). Gender trouble. Routledge.

Chen, D., Strang, J. F., Kolbuck, V. D., Rosenthal, S. M., Wallen, K., Waber, D. P., Steinberg, L., Sisk, C. L., Ross, J., Paus, T., Mueller, S. C., McCarthy, M. M., Micevych, P. E., Martin, C. L., Kreukels, B. P. C., Kenworthy, L., Herting, M. M., Herlitz, A., Hebold Haraldsen, I. R. J., ... Garofalo, R. (2020). Methodologies to evaluate neurodevelopmental effects of pubertal suppression in transgender youth. Transgender Health, 5(4), 246-257. https:// doi.org/10.1089/trgh.2020.0006

Clemens, B., Junger, J., Pauly, K., Neuschaefer-Rube, C., Frölich, D., Mingoia, G., Derntl, B., \& Habel, U. (2017). Male-to-female gender dysphoria: Gender-specific differences in resting-state networks. Brain and Behavior, 7(5). https://doi.org/10.1002/ brb3.691

Cohen-Kettenis, P. T. (2005). Gender change in 46, XY persons with 5 alpha-reductase-2 deficiency and 17beta-hydroxysteroid dehydrogenase-3 deficiency. Archives of Sexual Behavior, 34 , 399-410. https://doi.org/10.1007/s10508-005-4339-4

Dessens, A. B., Slijper, F. M., \& Drop, S. L. (2005). Gender dysphoria and gender change in chromosomal females with congenital adrenal hyperplasia. Archives of Sexual Behavior, 34, 389-397. https://doi.org/10.1007/s10508-005-4338-5

Drabant, E. M., Kiefer, A. K., Eriksson, N., Mountain, J. L., Francke, U., Tung, J. Y., Hinds, D. A., \& Do, C. B. (2012). Genome-Wide Association Study of sexual orientation in a large, web-based cohort. 23andMe Inc.

Drummond, K. D., Bradley, S. J., Peterson-Badali, M., \& Zucker, K. J. (2008). A follow-up study of girls with gender identity disorder. Developmental Psychology, 44(1), 34-45. https://doi. org/10.1037/0012-1649.44.1.34

Feusner, J. D., Lindström, A., Moody, T. D., Dhejne, C., Bookheimer, S. Y., \& Savic, I. (2017). Intrinsic network connectivity and own body perception in gender dysphoria. Brain Imaging and Behavior, 11(4), 964-976. https://doi.org/10.1007/ s11682-016-9578-6

Fisher, A. D., Ristori, J., Castellini, G., Cocchetti, C., Cassioli, E., Orsolini, S., Sensi, C., Romani, A., Mazzoli, F., Cipriani, A., Ricca, V., Vignozzi, L., Viggiano, M. P., Mascalchi, M., Maggi, M., \& Gavazzi, G. (2020). Neural correlates of gender face perception in transgender people. Journal of Clinical Medicine, 9(6), 1731. https://doi.org/10.3390/jem9061731

Folkierska-Żukowska, M., Rahman, Q., Marchewka, A., Wypych, M., Droździel, D., Sokołowski, A., \& Dragan, W. Ł. (2020). Male sexual orientation, gender nonconformity, and neural activity during mental rotations: an fMRI study. Science and Reports, 10(1), 18709. https://doi.org/10.1038/s41598-020-74886-0

Garcia-Falgueras, A., \& Swaab, D. F. (2008). A sex difference in the hypothalamic uncinate nucleus: Relationship to gender identity. Brain, 131(12), 3132-3146. https://doi.org/10.1093/brain/ awn276

Giedd, J., Vaituzis, A., Hamburger, S., Lange, N., Rajapakse, J., Kaysen, D., Vauss, Y., \& Rapoport, J. (1996). Quantitative MRI of the temporal lobe, amygdala, and hippocampus in normal human 
development: Ages 4-18 years. Journal of Comparative Neurology, 366(2), 223-230. https://doi.org/10.1002/(SICI)10969861(19960304)366:2\%3c223::AID-CNE3\%3e3.0.CO;2-7

Gizewski, E. R., Krause, E., Schlamann, M., Happich, F., Ladd, M. E., Forsting, M., \& Senf, W. (2009). Specific cerebral activation due to visual erotic stimuli in male-to-female transsexual compared with male and female controls: An fMRI study. Journal of Sexual Medicine, 6(2), 440-448. https://doi.org/10.1111/j.1743-6109. 2008.00981.x

Green, R. (2000). Family co-occurrence of "gender dysphoria": Ten sibling or parent-child pairs. Archives of Sexual Behavior, 29(5), 499-507. https://doi.org/10.1023/a:1001947920872

Guillamon, A., Junque, C., \& Gómez-Gil, E. (2016). A review of the status of brain structure research in transsexualism. Archives of Sexual Behavior, 45(7), 1615-1648. https://doi.org/10.1007/ s10508-016-0768-5

Hahn, A., Kranz, G. S., Küblböck, M., Kaufmann, U., Ganger, S., Hummer, A., Seiger, R., Spies, M., Winkler, D., Kasper, S., Windischberger, C., Swaab, D. F., \& Lanzenberger, R. (2015). Structural connectivity networks of transgender people. Cerebral Cortex, 25(10), 3527-3534. https://doi.org/10.1093/cercor/bhu194

Herbert, J. (2008). Who do we think we are? The brain and gender identity. Brain, 131(12), 3115-3117. https://doi.org/10.1093/ brain/awn 257

Heylens, G., De Cuypere, G., Zucker, K. J., Schelfaut, C., Elaut, E., Vanden Bossche, H., De Baere, E., \& Tsjoen, G. (2012). Gender identity disorder in twins: A review of the case report literature. Journal of Sexual Medicine, 9(3), 751-757. https://doi.org/10. 1111/j.1743-6109.2011.02567.x

Hines, M. (2004). Brain gender. Oxford University Press.

Hoekzema, E., Schagen, S. E., Kreukels, B. P., Veltman, D. J., CohenKettenis, P. T., Delemarre-van de Waal, H., \& Bakker, J. (2015). Regional volumes and spatial volumetric distribution of gray matter in the gender dysphoric brain. Psychoneuroendocrinology, 55, 55-59. https://doi.org/10.1016/j.psyneuen.2015.01.016

Hu, S., Wang, Q., Xu, Y., Liao, Z. L., Xu, L. J., Xu, X. J., Wei, E. Q., Yan, L. Q., Hu, J. B., Wei, N., Zhou, W. H., Huang, M. L., \& Zhang, M. M. (2011). Haemodynamic brain response to visual stimuli is different between homosexual and heterosexual men. Journal of International Medicine Research, 39(1), 199-211

Hu, S., Wei, E. Q., Wang, Q., Yan, L. Q., Wei, E. Q., Zhang, M. M., Hu, J. B., Huang, M. L., Zhou, W. H., \& Xu, Y. (2008). Patterns of brain activation during visually evoked sexual arousal differ between homosexual and heterosexual men. American Journal of Neuroradiology, 29(10), 1890-1896. https://doi.org/10.3174/ ajnr.A1260

Hu, S., Xu, D., Peterson, B. S., Wang, Q., He, X., Hu, J., Xu, X., Wei, N., Long, D., Huang, M., Zhou, W., Xu, W., Zhang, M., \& Xu, Y. (2013). Association of Cerebral networks in resting state with sexual preference of homosexual men: A study of regional homogeneity and functional connectivity. PLOS ONE, 8(3), e59426. https://doi.org/10.1371/journal.pone.0059426

Hu, S., Xu, D., Peterson, B. S., Wang, Q., Lai, J., Hu, J., Wei, N., Zhang, M., \& Xu, Y. (2014). Differing default mode network activities in men with homosexual or heterosexual preferences. Journal of Sexual Medicine, 11(10), 2474-2484. https://doi.org/10.1111/ jsm. 12639

Joel, D., Berman, Z., Tavor, I., Wexler, N., Gaber, O., Stein, Y., Shefi, N., Pool, J., Urchs, S., Margulies, D., Liem, F., Hänggi, J., Jäncke, L., \& Assaf, Y. (2015). Sex beyond the genitalia: The human brain mosaic. Proceedings of the National Academy of Sciences, 112(50), 15468-15473. https://doi.org/10.1073/pnas.1509654112

Jordan-Young, R. M. (2010). Brainstorm: The flaws in the science of sex differences. Harvard University Press.

Jorge, J. C. (2010). The embryology of gender. Journal of LGBT Youth, 7(4), 310-319. https://doi.org/10.1080/19361653.2010.512519
Junger, J., Habel, U., Bröhr, S., Neulen, J., Neuschaefer-Rube, C., Birkholz, P., Kohler, C., Schneider, F., Derntl, B., \& Pauly, K. (2014). More than just two sexes: The neural correlates of voice gender perception in gender dysphoria. PLoS ONE, 9(11), e111672. https://doi.org/10.1371/journal.pone.0111672

Kagerer, S., Klucken, T., Wehrum, S., Zimmermann, M., Schienle, A., Walter, B., Vaitl, D., \& Stark, R. (2011). Neural activation toward erotic stimuli in homosexual and heterosexual males. Journal of Sexual Medicine, 8(11), 3132-3143. https://doi.org/10.1111/j. 1743-6109.2011.02449.x

Khorashad, B. S., Khazai, B., Talaei, A., Acar, F., Hudson, A. R., Borji, N., Saberi, H., Aminzadeh, B., \& Muellery, S. C. (2020). Neuroanatomy of transgender persons in a Non-Western population and improving reliability in clinical neuroimaging. Journal of Neuroscience Research, 98(11), 2166-2177. https://doi.org/10. 1002/jnr.24702

Kinnunen, L. H., Moltz, H., Metz, J., \& Cooper, M. (2004). Differential brain activation in exclusively homosexual and heterosexual men produced by the selective serotonin reputate inhibitor, fluoxetine. Brain Research, 1024(1-2), 251-254. https://doi.org/10.1016/j. brainres.2004.07.070

Koob, G. F., Everitt, B. J., \& Robbins, T. W. (2013). Reward, motivation and addition. In L. R. Squirre, D. Berg, F. E. Bloom, S. du Lac, A. Ghosh, \& N. C. Spitzer (Eds.), Fundamental neuroscience (4th ed., pp. 871-898). Elsevier.

Kranz, G. S., Hahn, A., Baldinger, P., Haeusler, D., Philippe, C., Kaufmann, U., Wadsak, W., Savli, M., Hoeflich, A., Kraus, C., Vanicek, T., Mitterhauser, M., Kasper, S., \& Lanzenberger, R. (2014a). Cerebral serotonin transporter asymmetry in females, males and male-to-female transsexuals measured by PET in vivo. Brain Structure and Function, 219(1), 171-183. https://doi.org/ 10.1007/s00429-012-0492-4

Kranz, G. S., Hahn, A., Kaufmann, U., Küblböck, M., Hummer, A., Ganger, S., Seiger, R., Winkler, D., Swaab, D. F., Windischberger, C., Kasper, S., \& Lanzenberger, R. (2014b). White matter microstructure in transsexuals and controls investigated by diffusion tensor imaging. Journal of Neuroscience, 34(46), 15466-15475. https://doi.org/10.1523/JNEUROSCI.2488-14.2014

Kranz, G. S., Hahn, A., Kaufmann, U., Tik, M., Ganger, S., Seiger, R., Hummer, A., Windischberger, C., Kasper, S., \& Lanzenberger, R. (2018). Effects of testosterone treatment on hypotalamic neuroplasticity in female-to-male transgender individuals. Brain Structure and Function, 223(1), 321-328. https://doi.org/10. 1007/s00429-017-1494-z

Kranz, G. S., Wadsak, W., Kaufmann, U., Savli, M., Baldinger, P., Gryglewski, G., Haeusler, D., Spies, M., Mitterhauser, M., Kasper, S., \& Lanzenberger, R. (2015). High-dose testosterone treatment increases serotonin transport biding in transgender people. Biological Psychiatry, 78(8), 525-533. https://doi.org/10. 1016/j.biopsych.2014.09.010

Kreukels, B. P. C., \& Guillamon, A. (2016). Neuroimaging studies in people with gender incongruence. International Review of Psychiatry, 28(1), 120-128. https://doi.org/10.3109/09540261.2015. 1113163

Kruijver, F. P. M., Zhou, J. N., Pool, C. W., Hofman, M. A., Gooren, L. J., \& Swaab, D. F. (2000). Male-to-female transsexuals have female neuron numbers in a limbic system. Journal of Clinical Endocrinology and Metabolism, 85, 2034-2041. https://doi.org/ 10.1210/jcem.85.5.6564

Ku, H. L., Lin, C. S., Chao, H. T., Tu, P. C., Li, C. T., Cheng, C. M., Su, T. P., Lee, Y. C., \& Hsieh, J. C. (2013). Brain signature characterizing the body-brain-mind axis of transsexuals. PLoS ONE, 8(7), e70808. https://doi.org/10.1371/journal.pone.0070808

Kurth, F., Jancke, L., \& Luders, E. (2016). Sexual dimorphism of Broca's region: More gray matter in female brains in Brodmann areas 
44 and 45. Journal of Neuroscience Research, 95(1-2), 626-632. https://doi.org/10.1002/jnr.23898

Lawrence, A. A. (2010). Sexual orientation versus age of onset as bases for typologies (subtypes) of gender identity disorder in adolescents and adults. Archives of Sexual Behavior, 38, 514-545. https://doi.org/10.1007/s10508-009-9594-3

LeVay, S. (1991). A difference in hypotalamic structure between heterosexual and homosexual men. Science, 253(5023), 1034-1037. https://doi.org/10.1126/science.1887219

Liao, Z., Patel, Y., Khairullah, A., Paker, N., \& Paus, T. (2021). Pubertal testosterone and the structure of the cerebral cortex in young men. Cerebral Cortex. https://doi.org/10.1093/cercor/bhaa389

Liberati, A. (2009). The PRISMA statement for reporting systematic reviews and meta-analysis of studies that evaluate healthcare interventions: explanation and elaboration. British Medical Journal, 339, b2700. https://doi.org/10.1136/bmj.b2700

Lin, C. S., Ku, H. L., Chao, H. T., Tu, P. C., Li, C. T., Cheng, C. M., Su, T. P., Lee, Y. C., \& Hsieh, J. C. (2014). Neural network of body representation differs between transsexuals and cissexuals. PLoS ONE, 9(1), e85914. https://doi.org/10.1371/journal.pone. 0085914

Lippa, R. A. (2002). Gender, nature, and nurture. Lawrence Erlbaum Associates.

Luders, E., Sánchez, F. J., Gaser, C., Toga, A. W., Narr, K. L., Hamilton, L. S., \& Vilain, E. (2009). Regional gray matter variation in maleto-female transsexualism. NeuroImage, 46(4), 904-907. https:// doi.org/10.1016/j.neuroimage.2009.03.048

Majid, D. S. A., Burke, S. M., Manzouri, A., Moody, T. D., Dhejne, C., Feusner, J. D., \& Savic, I. (2020). Neural systems for own-body processing align with gender identity rather than birth-assigned sex. Cerebral Cortex, 30(5), 2897-2909. https://doi.org/10.1093/ cercor/bhz282

Maney, D. L. (2016). Perils and pitfalls of reporting sex differences. Philosophical Transactions of the Royal Society B: Biological Sciences, 371(1688), 20150119

Manzouri, A., Kosidou, K., \& Savic, I. (2017). Anatomical and functional findings in female-to-male transsexuals: Testing a new hypothesis. Cerebral Cortex, 27(2), 998-1010. https://doi.org/ $10.1093 /$ cercor/bhv278

Manzouri, A., \& Savic, I. (2018). Cerebral sex dimorphism and sexual orientation. Human Brain Mapping, 39(3), 1175-1186. https:// doi.org/10.1002/hbm. 23908

Manzouri, A., \& Savic, I. (2019). Possible neurobiological underpinnings of homosexuality and gender dysphoria. Cerebral Cortex, 29(5), 2084-2101. https://doi.org/10.1093/cercor/bhy090

Matosin, N., Frank, E., Engel, M., Lum, J. S., \& Newell, K. A. (2014). Negativity towards negative results: A discussion of the disconnect between scientific worth and scientific culture. Disease Models \& Mechanisms, 7(2), 171-173. https://doi.org/10.1242/ dmm.015123

McFadden, D. (2002). Masculinization effects in the auditory system. Archives of Sexual Behavior, 31, 99-111. https://doi.org/10. 1023/A:1014087319682

Mohammadi, M. R., \& Khalegi, A. (2018). Transsexualism: A different viewpoint to brain changes. Clinical Psychopharmacology and Neuroscience, 16(2), 136-143. https://doi.org/10.9758/cpn. 2018.16.2.136

Moody, T. D., Feusner, J. D., Reggente, N., Vanhoecke, J., Holmberg, M., Manzouri, A., Khorashad, B. S., \& Savic, I. (2021). Predicting outcomes of cross-sex hormone therapy in transgender individuals with gender incongruence based on pre-therapy resting-state brain connectivity. Neuroimage Clinical, 29, 102517. https://doi. org/10.1016/j.nicl.2020.102517
Moser, C. (2010). Blanchard's autogynephilia theory: A critique. Journal of Homosexuality, 57(6), 790-809. https://doi.org/10.1080/ 00918369.2010 .486241

Mueller, S. C., De Cuypere, G., \& T'Sjoen, G. (2017). Transgender research in the 21st century: A selective critical review from a neurocognitive perspective. American Journal of Psychiatry, 174(12), 1155-1162. https://doi.org/10.1176/appi.ajp.2017. 17060626

Nawata, H., Ogomori, K., Tanaka, M., Nishimura, R., Urashima, H., Yano, R., Takano, K., \& Kuwabara, Y. (2010). Regional cerebral blood flow changes in female to male gender identity. Psychiatry \& Clinical Neuroscience, 64(2), 157-161. https://doi.org/10. 1111/j.1440-1819.2009.02059.x

Nota, N. M., Kreukels, B. P. C., den Heijer, M., Veltman, D. J., CohenKettenis, P. T., Burke, S. M., \& Bakker, J. (2017). Brain functional connectivity patterns in children and adolescent with gender dysphoria: Sex-atypical or not? Psychoneuroendocrinology, 86, 187-195. https://doi.org/10.1016/j.psyneuen.2017.09.014

Paul, T., Schiffer, B., Zwarg, T., Krüger, T. H., Karama, S., Schedlowski, M., Forsting, M., \& Gizewski, E. R. (2008). Brain response to visual sexual stimuli in heterosexual and homosexual males. Human Brain Mapping, 29(6), 726-735. https://doi.org/10.1002/ hbm.20435

Perry, D., Walder, K., Hendler, T., \& Shamay-Tsoory, S. G. (2013). The gender you are and the gender you like: Sexual preference and empathic neural response. Brain Research, 1534, 66-75. https:// doi.org/10.1016/j.brainres.2013.08.040

Pol, H. E. H., Cohen-Kettenis, P. T., van Haren, N. E. M., Peper, J. S., Brans, R. G. H., Cahn, W., Schnack, H. G., Gooren, L. J. G., \& Kahn, R. S. (2006). Changing your sex changes your brain; Influences of testosterone and estrogen on adult human brain structure. European Journal of Endocrinology, 155(1), S107-S114. https:// doi.org/10.1530/eje.1.02248

Ponseti, J., Granert, O., Jansen, O., Wolff, S., Mehdorn, H., Bosinski, H., $\&$ Siebner, H. (2009). Assessment of sexual orientation using the hemodynamic brain response to visual stimuli. Journal of Sexual Medicine, 6(6), 1628-1634. https://doi.org/10.1111/j.1743-6109. 2009.01233.x

Ponseti, J., Siebner, H., Klöppel, S., Wolff, S., Granert, O., Jansen, O., Mehdorn, H. M., \& Bosinski, H. A. (2007). Homosexual women have less grey matter in perirhinal cortex than heterosexual women. PLoS ONE, 2(8), e762. https://doi.org/10.1371/ journal.pone.0000762

Prendergast, D., Ardekani, B., Ikuta, T., John, M., Peters, B., DeRosse, P., Wellington, R., Malhotra, A., \& Szeszko, P. (2015). Age and sex effects on corpus callosum morphology across the lifespan. Human Brain Mapping, 36(7), 2691-2702. https://doi.org/10. $1002 / \mathrm{hbm} .22800$

Rametti, G., Carillo, B., Gómez-Gil, E., Junque, C., Segovia, S., Gomez, Á., \& Guillamon, A. (2011). White matter microstructure in female to male transsexuals before cross-sex hormonal treatment. A diffusion tensor imaging. Journal of Psychiatric Research, 45(2), 199-204. https://doi.org/10.1016/j.jpsychires.2010.05.006

Rametti, G., Carillo, B., Gómez-Gil, E., Junque, C., Zubiaurre-Elorza, L., Segovia, S., Gomez, Á., \& Guillamon, A. (2011). The microstructure of white matter in male to female transsexuals before cross-sex hormonal treatment. A DTI study. Journal of Psychiatric Research, 45(2), 949-954. https://doi.org/10.1016/j.jpsyc hires.2010.11.007

Rametti, G., Carrillo, B., Gómez-Gil, E., Junque, C., Zubiaurre-Elorza, L., Segovia, S., Gomez, Á., Karadi, K., \& Guillamon, A. (2012). Effects of androgenization on the white matter microstructure of female-to-male transsexuals. A diffusion tensor imaging study. Psychoneuroendocrinology, 37(8), 1261-1269. https://doi.org/ 10.1016/j.psyneuen.2011.12.019 
Roselli, C. E. (2018). Neurobiology of gender identity and sexual orientation. Journal of Neuroendocrinology, 30(7), e12562. https:// doi.org/10.1111/jne.12562

Safron, A., Klimaj, V., Sylva, D., Rosenthal, A. M., Li, M., Walter, M., \& Bailey, J. M. (2018). Neural correlates of sexual orientation in heterosexual, bisexual, and homosexual women. Scientific Reports, 8, 673. https://doi.org/10.1038/s41598-017-18372-0

Safron, A., Sylva, D., Klimaj, V., Rosenthal, A. M., Li, M., Walter, M., \& Bailey, J. M. (2017). Neural correlates of sexual orientation in heterosexual, bisexual, and homosexual men. Scientific Reports, 7, 41314. https://doi.org/10.1038/srep41314

Santarnecchi, E., Vatti, G., Déttore, D., \& Rossi, A. (2012). Intrinsic cerebral connectivity analysis in an untreated female-to-male transsexual subject: A first attempt using resting-state fMRI. Neuroendocrinology, 96(3), 188-193. https://doi.org/10.1159/ 000342001

Savic, I., \& Arver, S. (2011). Sex dimorphism of the brain in male-tofemale transsexuals. Cerebral Cortex, 21(11), 2525-2533. https:// doi.org/10.1093/cercor/bhr032

Savic, I., Berglund, H., \& Lindström, P. (2005). Brain response to putative pheromones in homosexual men. PNAS, 102(20), 7356-7361. https://doi.org/10.1073/pnas.0407998102

Savic, I., Garcia-Falgueras, A., \& Swaab, D. F. (2010). Sexual differentiation of the human brain in relation to gender identity and sexual orientation. Progress in Brain Research, 186, 41-62

Savic, I., \& Lindström, P. (2008). PET and MRI show differences in cerebral asymmetry and functional connectivity between homoand heterosexual subjects. Proceedings of the National Academy of Sciences, USA, 105(27), 9403-9408. https://doi.org/10.1073/ pnas.0801566105

Savin-Williams, R. C., \& Ream, G. L. (2007). Prevalence and stability of sexual orientation components during adolescence and young adulthood. Archives of Sexual Behavior, 36, 385-394. https://doi. org/10.1007/s10508-006-9088-5

Schöning, S., Engelien, A., Bauer, C., Kugel, H., Kersting, A., Roestel, C., Zwitserlood, P., Pyka, M., Dannlowski, U., Lehmann, W., Heindel, W., Arolt, V., \& Konrad, C. (2010). Neuroimaging differences in spatial cognition between men and male-to-female transsexuals before and during hormone therapy. Journal of Sexual Medicine, 7, 1858-1867. https://doi.org/10.1111/j.1743-6109. 2009.01484.x

Segal, N. L. (2006). Two monozygotic twin pairs discordant for femaleto-male transsexualism. Archives of Sexual Behavior, 35, 347 358. https://doi.org/10.1007/s10508-006-9037-3

Shah, N. M., Jessell, T. M., \& Sanes, J. R. (2012). Sexual difference of the nervous system. In E. R. Kandel, J. H. Schwartz, T. M. Jessell, S. A. Siegelbaum, \& A. J. Hudspeth (Eds.), Principles of neural science. (5th ed., pp. 1306-1327). McGraw-Hill Medical.

Simon, L., Kozák, L. R., Simon, V., Czobor, P., Unoka, Z., Szabó, Á., \& Csukly, G. (2013). Regional grey matter structure differences between transsexuals and healthy controls. A voxel based morphometry study. PLoS ONE, 8(12), e83947. https://doi.org/10. 1371/journal.pone.0083947

Skorska, M. N., Chavez, S., Devenyi, G. A., Patel, R., Thurston, L. T., Lai, M. C., Zucker, K. J., Chakravarty, M. M., Lobaugh, N. J., \& Van der Laan, D. P. (2021). A multi-modal MRI analysis of cortical structure in relation to gender dysphoria, sexual orientation, and age in adolescents. Journal of Clinical Medicine, 10(2), 345. https://doi.org/10.3390/jcm10020345

Smith, E. S., Junger, J., Derntl, B., \& Habel, U. (2015). The transsexual brain-A review of findings on the neural basis of transsexualism. Neuroscience and Biobehavioral Reviews, 59, 251-266. https:// doi.org/10.1016/j.neubiorev.2015.09.008

Soleman, R. S., Schagen, S. E., Veltman, D. J., Kreukels, B. P., CohenKettenis, P. T., Lambalk, C. B., Wouters, F., \& Delemarre-van de Waal, H. A. (2013). Sex differences in verbal fluency during adolescence: A functional magnetic resonance imaging study in gender dysphoric and control boys and girls. Journal of Sexual Medicine, 10(8), 1969-1977. https://doi.org/10.1111/jsm.12083

Spies, M., Hahn, A., Kranz, G. S., Sladky, R., Kaufmann, U., Hummer, A., Ganger, S., Kraus, C., Winkler, D., Seiger, R., Comasco, E., Windischberger, C., Kasper, S., \& Lanzenberger, R. (2016). Gender transition affects neural correlates of empathy: A resting state functional connectivity study with ultra high-field 7T MR imaging. NeuroImage, 138, 257-265. https://doi.org/10.1016/j neuroimage.2016.05.060

Starcevic, A., Dakovic, M., Radojicic, Z., \& Filipovic, B. (2020). A structural magnetic resonance imaging study in therapy naïve transsexual individuals. Folia Morphologica. https://doi.org/10. 5603/FM.a2020.0073

Swaab, D. F. (2007). Sexual differentiation of the brain and behavior. Best Practice \& Research Clinical Endocrinology \& Metabolism, 21(3), 1431-1444. https://doi.org/10.1016/j.beem.2007.04.003

Swaab, D. F. (2008). Sexual orientation and its basis in brain structure and function. Proceedings of the National Academy of Sciences, USA, 105(30), 10273-10274. https://doi.org/10.1073/pnas.08055 42105

Swaab, D. F., \& Hofman, M. A. (1990). An enlarged suprachiasmatic nucleus in homosexual men. Brain Research, 537(1-2), 141-148. https://doi.org/10.1016/0006-8993(90)90350-K

Sylva, D. (2013). Neural correlates of sexual arousal in heterosexual and homosexual women and men. Hormones and Behavior, 64(4), 673-684. https://doi.org/10.1016/j.yhbeh.2013.08.003

Uribe, C., Junque, C., Gómez-Gily, E., Abos, A., Mueller, S. C., \& Guillamon, A. (2020). Brain network interactions in transgender individuals with gender incongruence. Neuroimage, 211, 116613. https://doi.org/10.1016/j.neuroimage.2020.116613

Veale, J. F., Clarke, D. E., \& Lomax, T. C. (2010). Biological and psychological correlates of adult gender-variant identities: New findings. Personality and Individual Differences, 49(3), 252-257. https://doi.org/10.1016/j.paid.2010.03.045

Wang, D., Han, L., Xi, C., Xu, Y., Lai, J., Lu, S., Huang, M., Hu, J., Wei, N., Xu, W., Zhou, W., Lu, Q., He, H., \& Hu, S. (2020). Interactive effects of gender and sexual orientation on cortical thickness, surface area and gray matter volume: a structural brain MRI study. Quantitative Imaging in Medicine and Surgery, 10(4), 835-846. https://doi.org/10.21037/qims.2020.03.07

Wang, Z., Hu, J. B., Ji, G. J., Xu, D. R., Wang, D. D., Xi, C. X., Hu, C. C., Lu, J., Du, Y. L., Lu, Q. Q., Huang, T. T., Lai, J. B., Chen, J. K., Zhou, W. H., Wei, N., Xu, Y., Wang, K., \& Hu, S. H. (2020). Executive function and its relation to anatomical connectome in homosexual and heterosexual men. Quantitative Imaging in Medicine and Surgery, 10(10), 1973-1983. https://doi.org/10. 21037/qims-19-821b

Whiting, P., Rutjes, A. W., Reitsma, J. B., Bossuyt, P. M., \& Kleijnen, J. (2003). The development of QUADAS: a tool for the quality assessment of studies of diagnostic accuracy included in systematic reviews. BMC Medical Research Methodology, 25(3), 141-148. https://doi.org/10.1186/1471-2288-3-25

Wijchers, P. J., \& Festenstein, R. J. (2011). Epigenetic regulation of autosomal gene expression by sex chromosomes. Trends in Genetics, 27(4), 132-140. https://doi.org/10.1016/j.tig.2011.01.004

Witelson, S. F., Kigar, D. L., Scamvougeras, A., Kideckel, D. M., Buck, B., Stanchev, P. L., Bronskill, M., \& Black, S. (2008). Corpus callosum anatomy in right-handed homosexual and heterosexual men. Archives of Sexual Behavior, 37(6), 857-863. https://doi. org/10.1007/s10508-007-9276-y

Yokota, Y., Kawamura, Y., \& Kameya, Y. (2005). Callosal shapes at the midsagittal plane: MRI differences of normal males, normal females, and GID. In Proceedings of the 27th annual conference of IEEE Engineering in Medicine and Biology, September 1-4, Shanghai, China. https://doi.org/10.1109/IEMBS.2005.1617119 
Zeki, S., \& Romaya, J. P. (2010). The brain reaction to viewing faces of opposite- and same-sex romantic partners. PLoS ONE, 5(12), e15802. https://doi.org/10.1371/journal.pone.0015802

Zhang, T. Y., \& Meaney, M. J. (2010). Epigenetics and the environmental regulation of the genome and its function. Annual Review of Psychology, 61, 439-466. https://doi.org/10.1146/annurev.psych. 60.110707 .163625

Zhou, J. N., Hofman, M. A., Gooren, L. J., \& Swaab, D. F. (1995). A sex difference in the human brain and its relation to transsexuality. Nature, 378(6552), 68-70. https://doi.org/10.1038/ $378068 \mathrm{a} 0$

Zubiaurre-Elorza, L., Junque, C., Gómez-Gil, E., Segovia, S., Carrillo, B., Rametti, G., \& Guillamon, A. (2013). Cortical thickness in untreated transsexuals. Cerebral Cortex, 23(12), 2855-2862. https://doi.org/10.1093/cercor/bhs267

Zucker, K. J., Bradley, S. J., Oliver, G., Blake, J., Fleming, S., \& Hood, J. (1996). Psychosexual development of women with congenital adrenal hyperplasia. Hormones and Behavior, 30(4), 300-318. https://doi.org/10.1006/hbeh.1996.0038

Publisher's Note Springer Nature remains neutral with regard to jurisdictional claims in published maps and institutional affiliations. 